\title{
Calidad del servicio administrativo: impacto sobre el compromiso, la satisfacción y el rendimiento de estudiantes universitarios
}

\author{
Javier Quesada*
}

Fecha de recibido: 8 de marzo de 2021 Fecha de aprobado: 8 de octubre de 2021

Para citar este artículo: Quesada, J. (2021). Calidad del servicio administrativo: impacto sobre el compromiso, la satisfacción y el rendimiento de estudiantes universitarios. Revista Universidad E Empresa, 23(41), 1-42. https://doi.org/10.12804/ revistas.urosario.edu.co/empresa/a.10352

\section{Resumen}

El presente artículo pretende comprender la relación que existe entre la calidad del servicio administrativo, la satisfacción, el compromiso y el rendimiento de los estudiantes universitarios, en procura de una visión desde la perspectiva latinoamericana. Se investigó el impacto que tiene esta calidad en una población de estudiantes del área de ciencias básicas de la Universidad de Costa Rica. Para ello se empleó un esquema mixto de investigación (cuantitativa-cualitativa) para contrastar la información obtenida. Se determinó que el estudiantado considera que la calidad del servicio impacta su satisfacción, pero no así el compromiso. Además, ninguno de los aspectos anteriores tiene influencia sobre su rendimiento. Se identificó que las personas estudiantes entienden que la satisfacción es producto del servicio académico y que el compromiso se asocia a otros elementos en el contexto universitario. Finalmente, el rendimiento aparece desvinculado a los aspectos estudiados.

Palabras clave: calidad; servicio; compromiso; satisfacción; rendimiento; universidad.

Máster en Administración y Química Industrial por la Universidad de Costa Rica (San José, Costa Rica). Filiación: Escuela de Química, Universidad de Costa Rica, y doctorando en Administración en la Universidad Estatal a Distancia. Docente en la Universidad de Costa Rica (San José, Costa Rica). Correo electrónico: francisco.quesada@ucr.ac.cr ORCID: https://orcid. org/0000-0003-2358-5809 


\title{
Administrative Service Quality: Impact on the Commitment, Satisfaction, and Performance of University Students
}

\begin{abstract}
This paper aims to present the understanding of the relationship between administrative quality service and satisfaction, engagement, and performance of higher education students in the Latin American context. Researcher studied the impact of quality in natural science students at the University of Costa Rica, using a mixed method (quantitative-qualitative) to compare the information. Researcher found that quality service impacts satisfaction but not engagement. None of the above have an impact on performance. It was possible to identify that those students understand satisfaction as a consequence of academic service, while engagement is relative to the other elements of the university context. Finally, performance is unrelated to the aspects studied.
\end{abstract}

Keywords: Quality; service; engagement; satisfaction; performance; university.

\section{Qualidade do serviço administrativo: impacto no comprometimento, na satisfação e no rendimento de estudantes universitários}

\section{Resumo}

Este artigo tem como objetivo compreender a relação existente entre a qualidade do serviço administrativo, a satisfação, o comprometimento e o rendimento de estudantes universitários, buscando uma visão na perspectiva latino-americana. Investigou-se o impacto da qualidade desse serviço em uma população de estudantes da área de ciências básicas da Universidade da Costa Rica. Para isso, um esquema de pesquisa misto (quantitativo-qualitativo) foi utilizado para contrastar as informações obtidas. Determinou-se que os alunos consideram que a qualidade do serviço administrativo impacta na sua satisfação, mas não no seu comprometimento. Além disso, nenhum dos aspectos anteriores influencia o seu rendimento. Identificou-se que os alunos entendem que a satisfação é produto do serviço acadêmico e que o comprometimento está associado a outros elementos do contexto universitário. Por fim, o rendimento parece não estar relacionado aos aspectos estudados.

Palavras-chave: qualidade; serviço; compromisso; satisfação; rendimento; universidade.

\section{Introducción}

La calidad es un concepto que ha permeado la sociedad y que se ha tornado un elemento esencial en la toma de decisiones, generalmente de compra. Es un término surgido de la industria dedicada a la producción de bienes, por lo que su desarrollo se produjo en un contexto de parámetros definidos cuantitativamente. Los consumidores se han enfocado en la calidad, piensan que entienden lo que significa, que la reconocen al verla y demandan calidad en cada producto (Hoyer \& Hoyer, 2001). La mayoría usamos el término 'calidad' de una manera que consideramos tiene sentido y es entendible para los demás, sin embargo, la definición es más compleja de lo que inicialmente parece. 
Solo el hecho de establecer criterios 'homogéneos' para objetivar su valoración ha sido tema de debate intenso en el mundo académico e industrial. El calificativo a un producto de 'alta o buena calidad' está asociado, en la mayoría de los casos, a percepciones por parte del usuario. Probablemente sea un concepto muy diferente si se evalúa desde la óptica de la persona u organización que invierte su esfuerzo y recursos en lograr hacerlo y ofrecerlo al público. Esta divergencia de puntos de vista ha llevado a la International Organization for Standardization (ISO) a delimitar el concepto a características que satisfagan los requerimientos de las partes interesadas. Esto sin duda establece un panorama complejo, ya que incorpora clientes directos, organizaciones que velan por el consumidor, los estados y los mismos productores del bien.

El escenario se torna más complicado cuando la calidad se extrapola al área de servicios. La estandarización de los procesos se vuelve más difícil por involucrar mayor intervención del factor humano. Si en el caso de bienes la valoración objetiva ha sido un trabajo laborioso, en el campo de los servicios la subjetividad propia de los actores en el proceso torna la evaluación aún menos objetiva y sumamente variable. No obstante, las empresas fueron las primeras en verse obligadas a incursionar en el área, por el concepto que ya los consumidores del servicio habían incorporado producto del acercamiento realizado por la industria dedicada a la producción de bienes.

El progreso en la valoración y mejoramiento de la calidad en los servicios ha resultado un proceso más lento que el efectuado para bienes, a pesar de la experiencia previa que se tuvo con este segundo. Los servicios, por su naturaleza de intangibilidad y de inmediatez de su elaboración y consumo, se han tornado un sistema que se encasilla y se hace calzar en las estructuras organizativas denominadas newtonianas predominantes en la filosofía industrial posterior al modelo desarrollado por Ford. Los mecanismos de causa-efecto resultan muy rígidos para procesos tan flexibles como los desarrollados en el ámbito de los servicios.

Las empresas que lucran con la prestación de servicios fueron las primeras en incursionar en el tema de la calidad. Razones obvias de sostenibilidad de ingresos y posición en el mercado marcaron el inicio de una tendencia. Sin embargo, las organizaciones no lucrativas también observaron oportunidades de mejora, producto de iniciativas propias o promovidas por la competencia privada o por atender a usuarios más sensibilizados por la cultura de calidad emprendida por las empresas. 
En este marco es que la educación inicia su etapa de evaluación de la calidad de los servicios que brinda. En una primera instancia se consideró la evaluación de la calidad de la formación que brindaban, en especial en las universidades de origen privado. Estas mediciones se fundamentaron en aspectos cuantitativos por medio de indicadores de colocación de egresados en el mercado laboral, resultados en exámenes estandarizados, evaluaciones de entes externos especializados en el tema, gremios profesionales, entre otros (EsIB-The National Unions of Students in Europe, 2002).

No obstante, la calidad se valoraba en términos de la excelencia académica del estudiante. No se consideraba el servicio de educación como un producto de la suma de una serie de subservicios internos. Esto lo empezaron a reconocer las organizaciones de educación superior e implementaron las primeras medidas para evaluar la calidad del servicio denominado no académico. Las más importantes aproximaciones son extrapolaciones de modelos de medición provenientes del marketing de servicios, con adaptaciones al tipo de organización que son las universidades.

Las universidades públicas se han enfrentado a la necesidad de incorporar también dentro de sus sistemas de aseguramiento de la calidad rúbricas que contemplan los servicios. La mayoría se efectúan con enfoques cuantitativos por tratarse de acoplamientos de modelos preexistentes, aunque también se observan algunos acercamientos cualitativos, pero con enfoques muy dispares que producen resultados según la arista valorada. Una orientación frecuente es el impacto sobre el rendimiento del estudiantado o la implicación que tiene ese servicio interno, generalmente denominado administrativo, en la permanencia en el centro educativo. En estas aproximaciones se parte de la premisa de que el estudiante es el cliente central de la educación.

El rendimiento no es el único componente que se afecta por el servicio administrativo y podría ser un reflejo del efecto sobre otros factores como la satisfacción y el compromiso. La relación entre los diferentes elementos y el servicio administrativo es un tema poco explorado. Sin embargo, los trabajos reportados se podrían dividir en tres tipos:

- Relación entre la calidad del servicio y la satisfacción: las investigaciones se han orientado a valorar la relación que existe entre estos dos elementos. Los servicios a los que se presta énfasis son diferentes. Los resultados son muy variados 
y se brinda peso desigual a los componentes según la población estudiada y el país en que se realiza (Arslanagic-Kalajdzic et al., 2014; Campos et al., 2017; Candelas Zamorano et al., 2013; Eberle et al., 2016; Hemsley-Brown et al., 2010). Algunas otras investigaciones valoran la relación simultánea entre calidad de servicio administrativo y satisfacción para la población estudiantil y docente (Loureiro \& González, 2012; Navarro et al., 2005; Torres \& Araya, 2010).

- Relación de la calidad del servicio y el compromiso: la investigación en esta línea es significativamente inferior. Se presentan trabajos aislados que no reflejan una profundización en el vínculo entre los dos elementos (Krause \& Coates, 2008; Pineda Báez et al., 2014).

- No se identifican estudios de la relación calidad del servicio y el rendimiento del estudiantado, pero sí algunos relacionados con el efecto del compromiso y satisfacción sobre este (Ahmed et al., 2010; Salgado, 2013).

Prácticamente la totalidad de los estudios se efectúa con un enfoque predominantemente cuantitativo. Una combinación del enfoque cuantitativo y cualitativo abre la posibilidad para obtener visiones complementarias del fenómeno por estudiar, las cuales ayuden a comprender de manera integral los impactos que tiene una gestión administrativa con o sin estándares de calidad acordes con las exigencias de las instituciones académicas actuales. Sobre todo porque se plantea desde la perspectiva de captar las impresiones del cliente primario de la educación: el estudiante.

La presente propuesta constituye una primera aproximación al tema de la calidad del servicio administrativo y su relación con la satisfacción, el compromiso y el rendimiento de los estudiantes en la educación superior latinoamericana de manera integral. Permite tener una mirada inicial a cómo el modelo de universidad pública impacta sobre el cliente directo que tiene. 


\section{Revisión de literatura e hipótesis}

En el marco de esta investigación es relevante revisar los aspectos conceptuales que soportan los elementos de interés y la forma en que se realiza la aproximación a su medición desde el punto de vista cuantitativo.

\subsection{La calidad en los servicios}

La calidad en los servicios tiene que tratarse de manera diferente porque no puede evaluarse y diagnosticarse de la misma forma que cuando se habla de manufactura. Esto motivó el desarrollo de técnicas particulares para abarcar el tema de la calidad en los servicios (Tumino \& Poitevin, 2014) basados en el modelo SERvQual, y se incorporaron ítems que atienden a otros aspectos del servicio universitario tales como los que proponen Rubio Gómez, Aguilar Feijoo, Massa Sánchez, Maldonado y Ramírez Asanza (2005). Existen características particulares que diferencian los servicios de los productos de manufactura. Algunos autores hacen referencia a las siguientes:

- La intangibilidad: la mayoría de los servicios son intangibles. No es posible para el cliente tener un criterio previo a haber recibido el servicio (Duque Oliva, 2005; Matsumoto Nishizawa, 2014; Parasuraman et al., 1985; Tumino \& Poitevin, 2014).

- La inseparabilidad: la producción y consumo del servicio es de manera simultánea, se realizan al mismo tiempo (Duque Oliva \& Diosa Gómez, 2014; Parasuraman et al., 1985; Tumino \& Poitevin, 2014).

- La heterogeneidad: los resultados de la prestación del servicio pueden ser diferentes, afectando la calidad y su evaluación (Duque Oliva \& Diosa Gómez, 2014; Matsumoto Nishizawa, 2014; Parasuraman et al., 1985; Tumino \& Poitevin, 2014). 
- Carácter perecedero: el servicio se extingue en el momento de ser consumido por el cliente. No es posible almacenarlo o devolverlo (Duque Oliva, 2005; Tumino \& Poitevin, 2014).

- También se incluyen el contacto con el cliente y la relación basada en el cliente como caracterizadores (Duque Oliva \& Diosa Gómez, 2014).

Dadas las características señaladas, la calidad del servicio está relacionada con una percepción del desempeño. Este es el fundamento de la catalogación del servicio con base en las expectativas del cliente sobre un intangible que no puede ser valorado previamente. Dentro de este contexto, el concepto hace referencia a una calidad percibida que, por lo tanto, es subjetiva y va más allá de los atributos del producto. Como consecuencia, la calidad percibida del servicio no puede ser tratada de la misma forma que la calidad de bienes tangibles (Duque Oliva \& Diosa Gómez, 2014; Eberle et al., 2016).

\subsubsection{Medición de la calidad en el servicio: una percepción cuantificada}

El desarrollo de la calidad del servicio ha sido un tópico de gran interés para los administradores de negocios. Inherentemente se ha trabajado en modelos que sirvan para cuantificarla. Esencialmente han sido generados en Europa, Estados Unidos y otros países desarrollados durante los últimos 35 años (Galeeva, 2016). En la tabla 1 se resumen los principales modelos descritos en la literatura.

El modelo más empleado es el Servqual (service quality). El modelo cuenta con cinco dimensiones y se mide la brecha entre el rendimiento y las expectativas que se tienen. En su versión original está constituido por 22 ítems que se valoran en una escala de Likert de siete puntos (Bayraktaroglu \& Atrek, 2010).

Parasuraman, Zeithaml y Berry (1988) definen las dimensiones valoradas en el Servqual de la siguiente forma:

- Tangibilidad: instalaciones físicas, equipo y apariencia del personal. 
- Confiabilidad: capacidad para realizar el servicio prometido de manera confiable y exacta.

- Capacidad de respuesta: disposición a ayudar al cliente y proveer el servicio solicitado.

- Seguridad: conocimiento y cortesía de los empleados y su habilidad para inspirar confianza y credibilidad.

- Empatía: interés y atención individualizada que la organización provee a sus clientes.

Tabla 1. Modelos implementados para la medición de la calidad del servicio

\begin{tabular}{ll}
\hline \multicolumn{1}{c}{ Modelo } & \multicolumn{1}{c}{ Descripción } \\
\hline Modelo de la imagen & $\begin{array}{l}\text { Desarrollado por la escuela nórdica y formulado por Grönroos, plantea que la calidad } \\
\text { percibida por los clientes es la interacción de la calidad técnica (qué se da) y la calidad } \\
\text { funcional (cómo se da), y su relación con la imagen corporativa. }\end{array}$ \\
\hline Modelo Servqual & $\begin{array}{l}\text { De la escuela estadounidense, Parasuraman, Zeithalml y Berry propusieron un ins- } \\
\text { trumento de medición mediante la evaluación por separado de las expectativas y }\end{array}$ \\
& percepciones de un cliente. Es el más utilizado por los investigadores. \\
\hline Modelo de los tres compo- & El modelo incluye los siguientes elementos: el servicio y sus características, el proceso \\
& de envío o entrega del servicio, y el ambiente que rodea el servicio. Fue propuesto \\
& por Rust y Oliver. \\
\hline Modelo Servperf* & Se trata de una variación al Servqual que cambia el enfoque de la evaluación y las \\
& preguntas en los instrumentos. La propuesta fue realizada por Cronin y Taylor. \\
\hline Modelo de desempeño & Propuesto por Teas, el modelo sugiere puntuaciones ponderadas para el empleo del \\
evaluado (PE)* & Servqual.
\end{tabular}

* Varios de los autores consultados mencionan estos dos modelos de manera diferenciada al Servqual, sin embargo, son producto de modificaciones en la forma en que se realiza la medición.

Fuente: elaboración del autor con base en Bayraktaroglu y Atrek (2010), Cronin y Taylor (1992), Duque Oliva (2005), Eberle et al. (2016), Galeeva (2016), Lupo (2013) y Parasuraman et al. (1988).

El modelo se basa en las expectativas del cliente, que son creencias sobre el servicio que se entrega y funcionan como puntos de referencia para valorar el desempeño de la empresa u organización. La expectativa puede variar dependiendo de los estándares previos del cliente. Por otro lado, está la percepción, la cual se refiere a cómo valoran los clientes el servicio ofrecido y que se enlaza con las dimensiones descritas arriba para el Servqual (Matsumoto Nishizawa, 2014; Pereira Puga, 2014; Rezaei et al., 2017).

En el modelo Servperf, los autores parten del supuesto de que la expectativa siempre es la más alta y, por lo tanto, no se debe cuantificar. Sobre la base de esta premisa este 
modelo solamente valora la percepción de la calidad del servicio (Cronin \& Taylor, 1992). Hay estudios que sugieren que este modelo es mejor para medir la calidad, en especial cuando se trata de la educación superior (Bayraktaroglu \& Atrek, 2010; Duque Oliva, 2005; Galeeva, 2016).

\subsection{Satisfacción: el concepto}

Asociada estrechamente a la calidad se encuentra la satisfacción, la cual es un proceso que se inicia en el sujeto concreto y real, termina en este y, por lo tanto, es primordialmente subjetiva, desde su naturaleza hasta las técnicas de medición y de análisis. Sin embargo, en muchas ocasiones es necesario que otro efectúe alguna acción para que se cause un sentimiento de satisfacción o insatisfacción en el individuo. Entonces, esta no estaría dada por una percepción o circunstancia individual. Por el contrario, se trata de una sensación propia e incomparable de cada sujeto que está influenciada por un entorno y que genera un proceso intrasubjetivo e intersubjetivo complejo (Velandia et al., 2008).

El concepto ha ido evolucionando tras décadas de estudios al respecto. Se ha valorado desde aspectos como los atributos, confirmación de expectativas y los juicios de inquietud entre la satisfacción y las emociones generadas. La mayoría de los autores consideran que la satisfacción implica (Morales Sánchez \& Hernández Mendo, 2004):

1) La existencia de un objetivo que se desea alcanzar.

2) La consecución de este objetivo, evaluada contra una referencia de comparación.

3) El proceso de evaluación supone por lo menos un resultado y un estándar de comparación.

Para efectos del consumidor, la satisfacción se ha relacionado con la calidad del producto o servicio que le es ofrecido. Aquí se entra en el empleo de dos grandes grupos denominados clientes y usuarios (por ejemplo, el estudiantado). La diferencia básica radica en que los primeros desembolsan una cantidad de dinero para obtener lo que desean, mientras los segundos no. Hay que considerar que la satisfacción es una evaluación susceptible a ser modificada en cada transacción, mientras la calidad percibida es una evaluación más estable en el tiempo (Morales Sánchez \& Hernández Mendo, 2004). En el caso de los servicios, la calidad como 
actitud se actualiza en cada transacción, lo mismo que sucede con el rendimiento percibido y la satisfacción experimentada (Teas, 1993). En la línea de relación de calidad-satisfacción, la International Organization for Standardization (ISO, 2020) la define como "percepción del cliente sobre el grado en que se han cumplido sus requerimientos", donde el término cliente también abarca a los que antes se denominaron como usuarios.

\subsubsection{Mediación de la satisfacción estudiantil}

El primer documento estructurado para medir la satisfacción estudiantil fue desarrollado por la Universidad de Iowa en 1971 y se denominó College Student Satisfaction Questionnaire (cssQ). Este instrumento estaba constituido por 130 ítems que representaban seis dimensiones: políticas y procedimientos, condiciones de trabajo, compensación, calidad de educación, vida social y reconocimiento. Fue ajustado en 1977, 1982 y 1997 (Starr, Betz \& Menne, 1971).

Mejías y Martínez (2009) construyeron un cuestionario denominado Satisfacción Estudiantil Universitaria (sEu), que validaron en la Universidad de Carabobo en Venezuela. Nace sobre la base de cinco modelos de satisfacción de clientes y está conformado por cuatro dimensiones (enseñanza, organización académica, vida universitaria e infraestructura y servicios universitarios) con 52 ítems. Este cuestionario fue reducido a 19 ítems, pero siempre con las mismas cuatro dimensiones y con una valoración en una escala de Likert. La reducción del cuestionario es muy conveniente para que sea llenado por los participantes (Cadena Badilla, Mejías Acosta, Vega Robles \& Vásquez Quiroga, 2016).

También es popular un cuestionario de uso comercial denominado Student Satisfaction Inventory, distribuido por usa Group Noel-Levitz. Está conformado por 116 ítems y 11 dimensiones (efectividad del asesoramiento académico, clima del campus, vida en el campus, servicios de apoyo del campus, preocupación por el individuo, efectividad de instrucción, reclutamiento y efectividad de ayuda financiera, efectividad de inscripción, seguridad y protección en el campus, excelencia en el servicio y orientación al estudiante), que se valoran en una escala de Likert de 1 a 7 en relación con la satisfacción y la importancia en cada ítem, y se calcula una brecha entre las dos evaluaciones. 


\subsection{Compromiso: más que un enfoque sentimental}

Definir el concepto de compromiso debería ser algo relativamente sencillo, sin embargo, en la realidad existen muchos significados diferentes según el contexto y cómo se ha manejado por la organización (Baron, 2012). Es por esta razón que el término se define en función de la situación particular que se desee analizar y se tiene que desmarcar del discurso romántico y muchas veces populista con que se trata por muchas organizaciones de todo tipo.

El constructo de compromiso estudiantil tiene varios conceptos. Una de las aproximaciones lo define como el grado de atención, curiosidad, interés, optimismo y pasión de los estudiantes cuando están aprendiendo o han aprendido (Great Schools Partnership, 2018). La National Survey of Student Engagement (NSSE) considera que se trata de la combinación de la cantidad de tiempo y esfuerzo que ponen los estudiantes en el estudio y otras actividades con propósitos educativos (NSSE, 2018). Gallup (citado por Olson y Peterson, 2018) menciona que el término es usado para describir un interés individual y entusiasmo por la universidad que impacta en su rendimiento académico y comportamiento.

Otros autores definen el término como una representación de la calidad del esfuerzo e involucramiento en actividades productivas para el aprendizaje (Abdullah et al., 2015; Kuh, 2009). También se usa la definición de que es la participación activa e informada de estudiantes en formar y desarrollar su experiencia estudiantil (Leeds Trinity University, 2018). A esta definición Trowler (2010) añade que se cuente con resultados medibles. La principal organización que trabaja en la medición del compromiso estudiantil, la NSse, incorpora el aporte de la institución en recursos, organización del currículum y otras oportunidades como componente importante.

Kahu (2013) expone varias perspectivas que son empleadas en la aproximación del concepto. Una de ellas es la de comportamiento, relacionada con la satisfacción y el logro del estudiante, incluido el tiempo en la tarea, la integración social y académica, y las prácticas de enseñanza. Para efectos prácticos este componente resulta difícil de medir y, por lo tanto, de valorar de forma precisa su importancia sobre el compromiso. Además, la definición dentro de la perspectiva del comportamiento es limitada y poco clara. Esto 
restringe su utilidad en la investigación para comprender el compromiso estudiantil. En muchas ocasiones se ha detectado que las prácticas institucionales influyen sobre el comportamiento de los estudiantes.

\subsubsection{Medición del compromiso estudiantil}

Los instrumentos desarrollados para medir este compromiso valoran múltiples aspectos. El interés ha sido generado del seno de centros de educación superior o de las autoridades responsables de la educación de algunos países. Los más empleados son: National Survey of Student Engagement (NSSE), que fue desarrollado en 1998 y aplicado en Estados Unidos y Canadá desde 2000, es un cuestionario que se compone de 106 ítems en total (Hardy \& Bryson, 2010; Pineda Báez et al., 2014). Otro muy empleado es el First Year Experience Questionnaire (FYEQ), que mide la retención de los estudiantes en el primer año universitario, aspecto importante en Australia (Hardy \& Bryson, 2010; Krause \& Coates, 2008). Así mismo, el National Student Survey (nss) es muy empleado en el Reino Unido como parte de la evaluación de la educación superior que está enfocada en medir el compromiso (Hardy \& Bryson, 2010; Ipsos MORI, 2018).

Es relevante señalar que este último instrumento se asemeja mucho al cuestionario que se emplea para medir la calidad por medio de la brecha de satisfacción (Servqual) que se describió en el apartado correspondiente a este tema. Adicionalmente, Schaufeli y colaboradores (2002) propusieron un instrumento denominado Utrecht Work Engagement Scale (uwEs), que está constituido por tres dimensiones. La primera de ellas es vigor (9 ítems), dedicación (8 ítems) y absorción (7 ítems). Una versión posterior redujo el número de ítems a 15, distribuidos equitativamente entre dimensiones (Schaufeli \& Bakker, 2003; Schaufeli et al., 2002; Schaufeli et al., 2006).

\subsection{Rendimiento académico estudiantil}

El rendimiento académico es una combinación de factores que influencian a la persona que aprende y que ha sido definido en términos de un valor que se asigna producto del cumplimiento de las tareas académicas. Tradicionalmente se mide mediante calificaciones cuantitativas que indican la aprobación o no de una materia. Las notas son un indicador 'objetivo' de 
los logros académicos de un estudiante que asume que incorpora los aspectos personales, académicos y sociales (Garbanzo, 2007).

Ese rendimiento académico, que también es denominado desempeño académico o logro académico y que en esencia se refieren a lo mismo, está establecido por cada universidad en dos niveles: nivel primario, que conlleva los criterios para la definición en el ámbito de cursos, y un segundo nivel, que es un promedio de las calificaciones obtenidas en las diferentes asignaturas. La forma en que se realiza esto es generalmente establecida por cada centro de educación superior.

El rendimiento académico se puede definir como "el producto final de la aplicación de su esfuerzo, mediatizado por actividades, rasgos y la percepción más o menos correcta de los cometidos asignados" (Salgado, 2013, p. 6). Hay consenso en lo difícil que resulta identificar este rendimiento en la educación superior solo con base en la calificaciones obtenidas por el estudiante, sin embargo, es lo usualmente empleado a pesar de las críticas ya planteadas desde hace varios años (Pérez Serrano, 1986).

El rendimiento académico se puede dividir en dos categorías por los resultados: inmediatos y mediatos. Para De Miguel y Arias (1999), estos se definen así:

Los primeros estarían determinados por los resultados/calificaciones que obtienen los alumnos a lo largo de los estudios hasta obtener la titulación correspondiente. Los segundos hacen referencia a la aplicación que la formación recibida por los titulados tiene en la vida social; es decir, la utilidad que dichos estudios tienen en el proceso de incorporación al mundo laboral de los graduados universitarios. Ambos criterios, también denominados rendimiento interno y externo, constituyen los parámetros de referencia que se emplean con mayor frecuencia para evaluar el rendimiento académico de la enseñanza superior. (p. 354)

Medir los resultados externos (mediatos) es complicado y laborioso, pues implica un seguimiento posterior de los graduados y las herramientas de medición son difíciles de estandarizar. Es más sencilla la medición de los resultados inmediatos, ya que están incorporados en la estructura de métricas de las instituciones de educación superior. Por ser multicausal 
implica distintos factores y espacios temporales que intervienen en el proceso de aprendizaje. Los aspectos que se asocian son de índole interna y externa al estudiante, y pueden ser de orden social, cognitivo y emocional (Garbanzo, 2007).

\subsection{1. ¿Cómo medir el rendimiento?}

Diferente a los constructos anteriores, para el rendimiento no se identifica un marco conceptual ni modelo que permita estructurar su medición. En el caso del rendimiento académico, la tradición ha dictado que se mida por las calificaciones que obtenga cada estudiante en los cursos que matricula, o bien como un progreso en la aprobación de las asignaturas que constituyen su malla curricular. Las metodologías para lograr establecer una calificación en un curso específico son variadas, sin embargo, convergen a un valor alfanumérico asociado a una escala de valoración (Newmann et al.,1996).

\subsection{Hipótesis de la investigación}

A partir de elementos expuestos anteriormente y de los trabajos llevados a cabo en esta temática es que se plantea la siguiente sospecha de investigación: El servicio administrativo tiene una influencia e impacto sobre la satisfacción, compromiso, y estos, a su vez, sobre el rendimiento de los estudiantes de la Facultad de Ciencias de la UCR.

Esto lleva a plantearse las siguientes hipótesis que atienden a las variables cuantitativas empleadas:

H1: Existe una correlación estadísticamente significativa entre la calidad del servicio administrativo y la satisfacción de los estudiantes.

H2: Existe una correlación estadísticamente significativa entre la calidad del servicio administrativo y el compromiso de los estudiantes.

H3: Existe una correlación estadísticamente significativa entre la calidad del servicio administrativo y el rendimiento de los estudiantes. 
H4: La calidad del servicio administrativo predice de forma estadísticamente significativa la satisfacción de los estudiantes.

H5: La calidad del servicio administrativo predice de forma estadísticamente significativa el compromiso de los estudiantes.

H6: La calidad del servicio administrativo predice de forma estadísticamente significativa el rendimiento de los estudiantes.

H7: La satisfacción del estudiantado predice de forma estadísticamente significativa su rendimiento.

H8: El compromiso del estudiantado predice de forma estadísticamente significativa su rendimiento.

H9: Los aspectos sociodemográficos moderan la calidad del servicio administrativo percibida, la satisfacción, compromiso y rendimiento de los estudiantes.

\section{Metodología}

La presente investigación es de tipo mixta porque utilizó técnicas con enfoque cuantitativo y cualitativo. El componente cuantitativo es de tipo expost facto. Esta es una metodología adecuada para establecer relaciones de causa-efecto y es una de las más empleadas en las ciencias sociales, económicas y administrativas. Por otro lado, la investigación cualitativa es de corte fenomenológica-hermenéutica, en el entendido de que se busca extraer sustantiva información empírica de un grupo de estudiantes respecto a su vivencia cotidiana y las percepciones sobre variables que conforman el objeto de estudio, y efectuar las interpretaciones de estas. 


\subsection{Muestra de estudio}

Los sujetos de estudio forman parte de tres (Biología, Geología y Química) de las cinco escuelas de la Facultad de Ciencias de la Universidad de Costa Rica. En el componente cuantitativo fue necesaria la aplicación de los cuestionarios. Los participantes representan diferentes niveles de avance en el plan de estudio. Se procuró mantener la misma relación que se presenta en la población para lo referente al sexo. Lo anterior también se aplicó en el grupo focal parte del componente cualitativo.

Para aplicar los cuestionarios correspondientes al componente cuantitativo, el número de participantes se estimó a partir de la fórmula para cálculo del tamaño de la muestra con conocimiento del tamaño de la población (Reinoso Sánchez, 2009):

$$
\mathrm{n}=\frac{Z_{\mathrm{a}}^{2} \mathrm{~N} p q}{(N-1) i^{2}+Z_{\mathrm{a}}^{2} p q}
$$

En donde:

$Z=$ nivel de confianza del 95\% (1.96)

$\mathrm{p}=$ probabilidad de éxito (que se responda el cuestionario) (0.50)

$q=$ probabilidad de fracaso (que no se responda el cuestionario) (0.50)

$\mathrm{N}$ = tamaño de la población (1091 estudiantes)

$\mathrm{n}=$ tamaño muestral

$\mathrm{i}=$ error de la estimación (10\%)

Con los valores especificados entre paréntesis, 88 personas estudiantes era el mínimo requerido. Sin embargo, el cuestionario fue contestado por 132 personas y esto disminuye el error a un $8 \%$. Las características sociodemográficas de este grupo se muestran 
en la tabla 2. La selección de ellas se efectuó mediante un muestreo no probabilístico por conveniencia. Los formularios fueron aplicados por medio de cuestionarios electrónicos enviados a los correos institucionales del estudiantado con una exhortación a participar. En el caso del grupo focal, las personas estudiantes fueron seleccionadas procurando una representatividad de tres niveles de avance en la carrera (inicial, medio, alto). Igualmente, se procuró el equilibrio de género y la representatividad según el número de estudiantes activos de cada escuela. Esta actividad se realizó por medio virtual.

Tabla 2. Características sociodemográficas de la población participante del estudio

\begin{tabular}{lcccccccccc}
\hline \multirow{2}{*}{ Característica } & \multicolumn{3}{c}{ Sexo } & \multicolumn{3}{c}{ Zona de origen } & \multicolumn{2}{c}{ Carrera } & \multicolumn{3}{c}{ Educación previa } \\
\cline { 2 - 12 } & Masculino & Femenino & Otro & Urbana & Rural & Biología & Geología & Química & Pública & Privada \\
\hline Individuos & 66 & 62 & 4 & 80 & 52 & 25 & 30 & 77 & 88 & 44 \\
\hline Porcentaje & 50.0 & 47.0 & 3.0 & 60.6 & 39.4 & 18.9 & 22.7 & 58.3 & 66.7 & 33.3 \\
\hline
\end{tabular}

Fuente: elaboración del autor.

\subsection{Instrumentos para recopilación de información}

Para el grupo focal se convocó a los estudiantes a llevar a cabo la actividad de manera virtual, la cual se ejecutó con la moderación del investigador. Se empleó una guía constituida por preguntas abiertas según las necesidades de información establecidas. Por otro lado, los cuestionarios empleados para hacer la investigación en su componente cuantitativo se elaboraron con base en los instrumentos estandarizados y validados internacionalmente para las dimensiones de calidad (Servperf), compromiso (uwes) y satisfacción (SEU).

El primer instrumento contempla en su constructo las escalas de tangible, capacidad de respuesta, confiabilidad, empatía y seguridad. En el caso del uwes, considera como escalas relevantes el vigor, la dedicación y la absorción. Finalmente, el instrumento aplicado para medir la satisfacción tiene como escalas aspectos académicos, aspectos administrativos, vida universitaria e infraestructura y servicios universitarios. Además, se incorporó una pregunta con respecto al autorrendimiento percibido. Los cuestionarios contemplan la valoración de las afirmaciones planteadas en una escala de Likert de 1 a 7 o de 0 a 6 . La pregunta sobre el rendimiento en una escala numérica de 1 a 10 . Adicionalmente, se incorporaron preguntas referentes a las características sociodemográficas de la población. Los cuestionarios empleados se muestran en los anexos 1, 2 y 3. 
Estos cuestionarios fueron elegidos por aspectos particulares en cada caso. Para evaluar la calidad, se eligió el Servperf, por ser el más empleado para esto, y además ha sido probado y validado en contextos académicos de nivel superior (Abdullah, 2006; Bayraktaroglu \& Atrek, 2010; Galeeva, 2016). Este instrumento responde al modelo de Servqual propuesto por Parasuraman y colaboradores, en el que se plantea que el paradigma expectativa-confirmación es el prevalente (Parasuraman et al., 1985, 1988). Por lo tanto, entre mayor sea la brecha entre lo esperado y la experiencia vivida, menor será la calidad percibida. Sin embargo, el instrumento empleado considera que la expectativa siempre es máxima.

En el caso del cuestionario uwes, fue empleado porque la literatura sugiere que este se ha usado con éxito en el ambiente de educación superior, aunado a que los instrumentos construidos por los países desarrollados valoran una perspectiva diferente del constructo compromiso objetivo de este estudio y que es producto del modelo antagónico al desarrollado por Maslach, que construyó su colega Schaufeli (Çapri et al., 2017; Römer, 2016; Schaufeli \& Bakker, 2003). En este caso el compromiso es uno de los extremos de un continuo en el que encontrarse con vigor, dedicarse a la labor encomendada y la absorción en la tarea son manifestaciones de un alto grado de compromiso, contrarias al desgano que se encuentra en el otro extremo.

Finalmente, el cuestionario seu se eligió porque fue desarrollado y validado en Latinoamérica, por lo que se consideró que se adapta mejor a las características de la población objeto de estudio (Buitrago-Suescún et al., 2017; Candelas Zamorano et al., 2013; Mejías \& Martínez, 2009). Este instrumento está planteado desde la perspectiva de la iso de la satisfacción, entendida como la satisfacción de los requerimientos del cliente. Al igual que la calidad, valora la brecha entre la expectativa y el rendimiento percibido del servicio, que tiene como consecuencia un nivel de ánimo alto (si la brecha es pequeña) o bajo (si la brecha es mayor).

La información recabada por medio de estos instrumentos constituye el eje angular para el componente cuantitativo de la investigación. Los datos suministrados por el estudiantado permiten realizar el análisis multivariante para tener información que se pueda contrastar con la que se obtiene del proceso de investigación cualitativo. Estos se constituyen por escalas provenientes del modelo conceptual al cual responden. 


\subsection{Análisis de los datos recopilados}

Por la naturaleza distinta de la información obtenida, esta se manejó según las técnicas propias de cada tipo de investigación, como se describe enseguida.

\subsubsection{Análisis de datos cualitativos}

Los grupos focales fueron grabados. Los audios fueron transcritos de forma fiel, pues se consideró que algunas reiteraciones y pausas eran importantes en la interpretación de los datos. Los documentos fueron analizados siguiendo el procedimiento sugerido por Seid (2016) y Hernández Sampieri y colaboradores (2010) para efectuar una codificación abierta definida de acuerdo con las categorías y subcategorías establecidas con anterioridad y otras emergentes. El criterio del investigador fue el que imperó en el proceso de la clasificación respectiva. Todas las transcripciones fueron analizadas y, a partir de esto, se efectuó una codificación axial y selectiva para pasar del nivel textual al nivel contextual con la ayuda del software Atlas.ti (Scientific Software Development GmbH, 2019). Finalmente, esta información fue sintetizada en un mapa semántico.

\subsubsection{Análisis de datos cuantitativos}

Los datos obtenidos de la aplicación de los cuestionarios se consolidaron en una hoja de cálculo y se realizó un curado de estos. Se identificaron y separaron las respuestas incompletas y las que contenían datos perdidos. Una vez depurados los datos, se procedió a transferirlos al software que se empleó. El análisis cuantitativo de los datos se efectuó empleando el software sPss® Statistics (IBM, 2015). Con este programa se procedió a la creación de las escalas para cada uno de los conceptos valorados en los cuestionarios aplicados. La confiabilidad de cada una de estas fue estimada calculando el coeficiente alfa de Cronbach factorial. Para el concepto de satisfacción, la escala 'vida universitaria' mostró un valor inferior a 0.60. Según la literatura, esta no debe ser considerada por tratarse de una escala que tiene una baja consistencia interna, es decir, los ítems que la conforman tienen una correlación pobre y se puede interpretar que no miden el constructo de interés (Ahdika, 2010; Streiner, 2003; Taber, 2017). 
Una vez corroborada la confiablidad de las escalas y con el propósito de analizar las potenciales correlaciones entre estas, se procedió a verificar el supuesto de comportamiento normal para decidir emplear el coeficiente de correlación de Pearson o de Spearman. Con tal fin primero se construyó un histograma para visualmente observar el comportamiento, luego se aplicaron las pruebas de Kolmogorov-Smirnov y Shapiro-Wilk. Adicionalmente, se construyeron gráficos Q-Q. En todas las pruebas efectuadas, los resultados indican que todas las escalas construidas presentan una tendencia no normal. Estos resultados conllevaron el empleo del coeficiente de correlación de Spearman para determinar el grado de asociación entre las escalas (variables).

En el caso de los modelos de regresión múltiple por el método paso a paso, que buscan encontrar la posible predicción por parte de alguna escala de otra, para cada uno de los resultados se realizaron gráficos P-P, histogramas, gráficos Q-Q y la prueba de Kolmogorov-Smirnov a fin de corroborar que se cumplía con los supuestos de normalidad que conlleva implícitamente el modelo de regresión multivariante. Además, se corroboró la homocedasticidad de las varianzas haciendo un gráfico de valor pronosticado versus residuo; y para verificar la independencia, se aplicó el contraste de Durbin-Watson. Las pocas regresiones que no cumplieron con los supuestos no fueron consideradas dentro del análisis.

El nivel de significancia estadística mínimo para todos los resultados presentados es de 0.05. Si hay mayor nivel de confianza (0.01), también se indicará al presentar los datos. Para el estudio de los efectos de moderación por aspectos sociodemográficos, donde se emplean valores nominales u ordinales, se emplearon modelos lineales generalizados (GLM, por su sigla en inglés), que precisamente deben ser usados para este tipo de valores. Aquí se consideraron los niveles de confianza ya citados. 


\section{Resultados}

Los análisis de correlación se llevaron cabo con base en el modelo conceptual de interacción propuesto, en el que la calidad impacta la satisfacción y compromiso de los estudiantes, y estas dos, su rendimiento. En la tabla 3 se observa cómo la correlación de las escalas de la calidad del servicio y las del compromiso es baja, moderada para el caso de la satisfacción, y no existe correlación entre la calidad del servicio y el rendimiento autopercibido. Estos resultados conducen a aceptar las hipótesis 1 y 2, y a rechazar la hipótesis 3 .

Los modelos de regresión lineal múltiple por pasos realizados para las escalas de satisfacción mostraron que la de aspectos administrativos, la de infraestructura y servicios universitarios, y la de aspectos académicos son predichas por algunas escalas de la calidad del servicio administrativo, como se muestra en la tabla 4. Los $\mathrm{R}$ cuadrados indican que en el modelo de tres pasos para la primera escala de satisfacción el $49 \%$ de la varianza es explicada por las escalas de calidad del servicio, mientras que un $43 \%$ lo hace para la segunda escala. Por su parte, el $37 \%$ de la varianza de los aspectos académicos es explicado por la calidad en un modelo de un paso. Con estos resultados se debe aceptar la hipótesis planteada número 4.

$\mathrm{Al}$ realizar el mismo ejercicio anterior para las escalas de compromiso no se identificaron modelos que fuesen estadísticamente significativos. Situación análoga se produjo cuando se procuró un modelo para el rendimiento, en el que no se pudo encontrar alguno. Por lo tanto, las hipótesis 5 y 6 son rechazadas. La regresión lineal múltiple para valorar la capacidad de predicción de las escalas del compromiso y la satisfacción del rendimiento del estudiantado mostraron que la satisfacción no lo predice, por lo que se rechaza la hipótesis 7. En el caso del compromiso, sí se encuentra un modelo en donde la escala de vigor predice en un $12 \%$ del $\mathrm{R}^{2}(\mathrm{~F}=18.28, \mathrm{p}<0.01)$ del rendimiento, con un coeficiente beta $=0.351(t=4.28, \mathrm{p}<0.01)$, por lo que la hipótesis 8 debería aceptarse, sin embargo, este valor es muy bajo, así que se rechaza. 
Tabla 3. Valores de correlación de Spearman entre la calidad del servicio administrativo y la satisfacción, compromiso y rendimiento del estudiantado

\begin{tabular}{|c|c|c|c|c|c|c|c|c|}
\hline & & \multicolumn{3}{|c|}{ Compromiso } & \multicolumn{3}{|c|}{ Satisfacción } & \multirow[b]{2}{*}{$\begin{array}{c}\text { Rendimiento } \\
\frac{0}{0} \\
\frac{0}{0} \\
\frac{0}{0} \\
\frac{0}{0} \\
\frac{0}{0} \\
\frac{7}{2} \\
\frac{1}{4}\end{array}$} \\
\hline \multicolumn{2}{|c|}{ Correlación de Spearman } & 흐 & 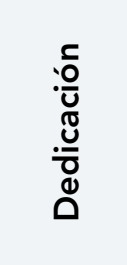 & 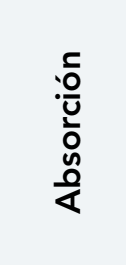 & 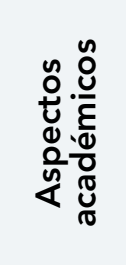 & 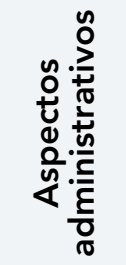 & 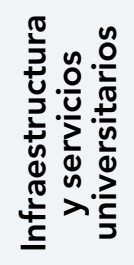 & \\
\hline \multirow{3}{*}{ Tangibles } & Coeficiente & $0.314^{\star \star}$ & $0.259^{\star \star}$ & $0.281^{\star \star}$ & $0.576^{\star \star}$ & $0.557^{\star \star}$ & $0.566^{\star \star}$ & 0.016 \\
\hline & Sig. (bilateral) & 0.000 & 0.003 & 0.001 & 0.000 & 0.000 & 0.000 & 0.858 \\
\hline & N & 132 & 132 & 132 & 132 & 132 & 132 & 132 \\
\hline \multirow{3}{*}{ Capacidad de respuesta } & Coeficiente & 0.163 & 0.148 & 0.088 & $0.460^{\star \star}$ & $0.578^{\star \star}$ & $0.401^{\star \star}$ & -0.012 \\
\hline & Sig. (bilateral) & 0.062 & 0.091 & 0.314 & 0.000 & 0.000 & 0.000 & 0.896 \\
\hline & N & 132 & 132 & 132 & 132 & 132 & 132 & 132 \\
\hline \multirow{3}{*}{ Confiabilidad } & Coeficiente & $0.216^{*}$ & $0.273^{\star \star}$ & $0.205^{\star}$ & $0.474^{\star \star}$ & $0.592^{\star \star}$ & $0.511^{\star \star}$ & 0.000 \\
\hline & Sig. (bilateral) & 0.013 & 0.002 & 0.019 & 0.000 & 0.000 & 0.000 & 0.996 \\
\hline & N & 132 & 132 & 132 & 132 & 132 & 132 & 132 \\
\hline \multirow{3}{*}{ Empatía } & Coeficiente & 0.250 ** & $0.212^{\star}$ & $0.222^{*}$ & $0.475^{\star \star}$ & $0.648^{\star \star}$ & $0.399 * \star$ & 0.018 \\
\hline & Sig. (bilateral) & 0.004 & 0.015 & 0.010 & 0.000 & 0.000 & 0.000 & 0.842 \\
\hline & N & 132 & 132 & 132 & 132 & 132 & 132 & 132 \\
\hline \multirow{3}{*}{ Seguridad } & Coeficiente & $0.185^{\star}$ & $0.189^{*}$ & $0.172^{*}$ & $0.481^{\star *}$ & $0.627^{\star \star}$ & $0.529 * \star$ & 0.015 \\
\hline & Sig. (bilateral) & 0.033 & 0.030 & 0.048 & 0.000 & 0.000 & 0.000 & 0.863 \\
\hline & N & 132 & 132 & 132 & 132 & 132 & 132 & 132 \\
\hline
\end{tabular}

* La correlación es significativa al nivel de 0.05 (bilateral).

** La correlación es significativa al nivel de 0.01 (bilateral).

Fuente: elaboración del autor.

Los efectos de moderación identificados son producto de la zona de procedencia del estudiante (urbana o rural) y su sexo. En las escalas de calidad del servicio se observa que la de tangibles es percibida de manera menos favorable por el estudiantado masculino que su contraparte femenina de zona rural, mientras los procedentes de zona urbana prácticamente no presentan diferencia entre sexo, como se ilustra en la figura 1a.

En el caso de la escala de empatía, la percepción difiere entre zonas y sexo. En la figura 1b se evidencia que para el estudiantado procedente de zona urbana el sexo masculino valora mejor esta escala, pero un comportamiento contrario se aprecia para el grupo que procede de zona rural. En la dimensión de satisfacción, la escala de aspectos académicos presenta un comportamiento marcadamente diferente para el estudiantado de zona rural, ya que 
el sexo masculino la valora menos favorablemente. Por el contrario, tanto hombres como mujeres tienen una valoración similar si su procedencia es urbana (figura 1c). Los resultados anteriores soportan la hipótesis 9.

Tabla 4. Modelos de regresión lineal múltiple para la calidad y satisfacción de los estudiantes

\begin{tabular}{|c|c|c|c|c|c|c|}
\hline \multirow[t]{3}{*}{ Variable } & \multicolumn{6}{|c|}{ Aspectos administrativos } \\
\hline & \multicolumn{2}{|c|}{ Paso 1} & \multicolumn{2}{|c|}{ Paso 2} & \multicolumn{2}{|c|}{ Paso 3} \\
\hline & b & $\mathbf{t}$ & b & $\mathbf{t}$ & b & $\mathbf{t}$ \\
\hline Seguridad & 0.654 & $9.86^{*}$ & 0.483 & $5.80 *$ & 0.327 & $3.07 *$ \\
\hline Tangibles & & & 0.269 & $3.24^{\star}$ & 0.210 & $2.45^{\star *}$ \\
\hline Empatía & & & & & 0.244 & $2.24^{\star \star}$ \\
\hline$R^{2}$ ( $R^{2}$ ajustado) & \multicolumn{2}{|c|}{$0.43(0.42)$} & \multicolumn{2}{|c|}{$0.47(0.46)$} & \multicolumn{2}{|c|}{$0.49(0.48)$} \\
\hline $\mathrm{F}$ & \multicolumn{2}{|c|}{97.21} & \multicolumn{2}{|c|}{57.38} & \multicolumn{2}{|c|}{41.20} \\
\hline $\mathrm{DR}^{2}$ & & & \multicolumn{2}{|c|}{0.04} & \multicolumn{2}{|c|}{0.02} \\
\hline \multicolumn{7}{|l|}{$N=132$} \\
\hline & \multicolumn{6}{|c|}{ Infraestructura y servicios universitarios } \\
\hline Tangibles & 0.580 & $8.11 *$ & 0.368 & $4.17^{\star}$ & 0.431 & $4.73^{\star}$ \\
\hline Seguridad & & & 0.332 & 3.75 & 0.498 & $4.39 *$ \\
\hline Empatía & & & & & -0.262 & $-2.29 * \star$ \\
\hline $\mathrm{R}^{2}$ (R² ajustado) & \multicolumn{2}{|c|}{$0.34(0.33)$} & \multicolumn{2}{|c|}{$0.40(0.39)$} & \multicolumn{2}{|c|}{$0.43(0.41)$} \\
\hline $\mathrm{F}$ & \multicolumn{2}{|c|}{65.86} & \multicolumn{2}{|c|}{43.29} & \multicolumn{2}{|c|}{31.54} \\
\hline $\mathrm{DR}^{2}$ & & & \multicolumn{2}{|c|}{0.06} & \multicolumn{2}{|c|}{0.03} \\
\hline
\end{tabular}

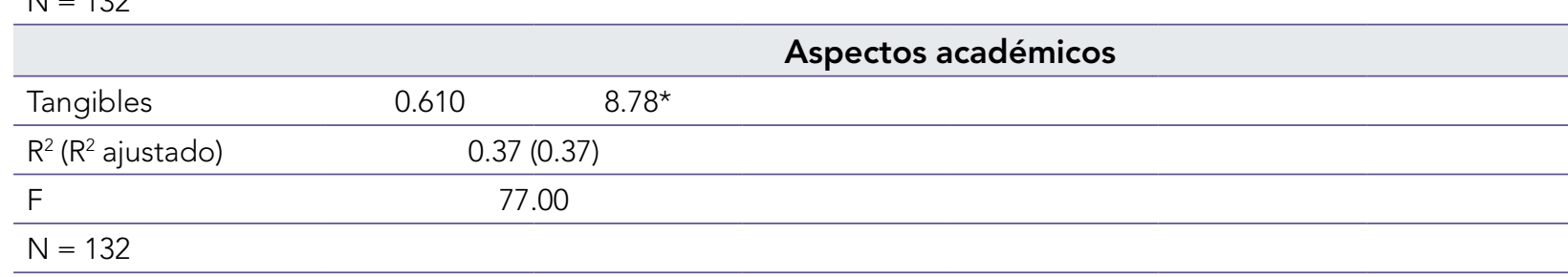

* La correlación es significativa al nivel de 0.05 (bilateral).

** La correlación es significativa al nivel de 0.01 (bilateral).

Fuente: elaboración del autor.

A partir del análisis de la información recabada en el grupo focal, se pudieron construir los mapas semánticos que se presentan en las figuras 2 y 3, referentes a los elementos que considera el estudiantado impactan su percepción del compromiso y la satisfacción producto del rol que tienen dentro del sistema universitario. 


\section{Discusión}

El modelo conceptual propuesto de un impacto directo de la calidad del servicio administrativo sobre la satisfacción y el compromiso del estudiantado, y, a su vez, de estos sobre el rendimiento, muestra un soporte en los resultados de correlación de Spearman. En la tabla 3 se puede observar que no existe una correlación entre el rendimiento y la calidad, pero sí para el compromiso (baja) y la satisfacción (moderada). Esto no significa que haya una causalidad entre los elementos mencionados, pero sí una tendencia en la misma dirección.

En los modelos predictivos se evidencia que la calidad explica la satisfacción del estudiantado, pero no su compromiso. La falta de esta causalidad se visualiza nuevamente en los resultados predictivos de los modelos de regresión, donde la satisfacción no predice el rendimiento y el compromiso lo hace en un nivel bastante bajo (12\%). De lo anterior se puede decir que el modelo propuesto no se apega a la realidad que muestra el estudio, pues este es como se ve en la figura 4. 

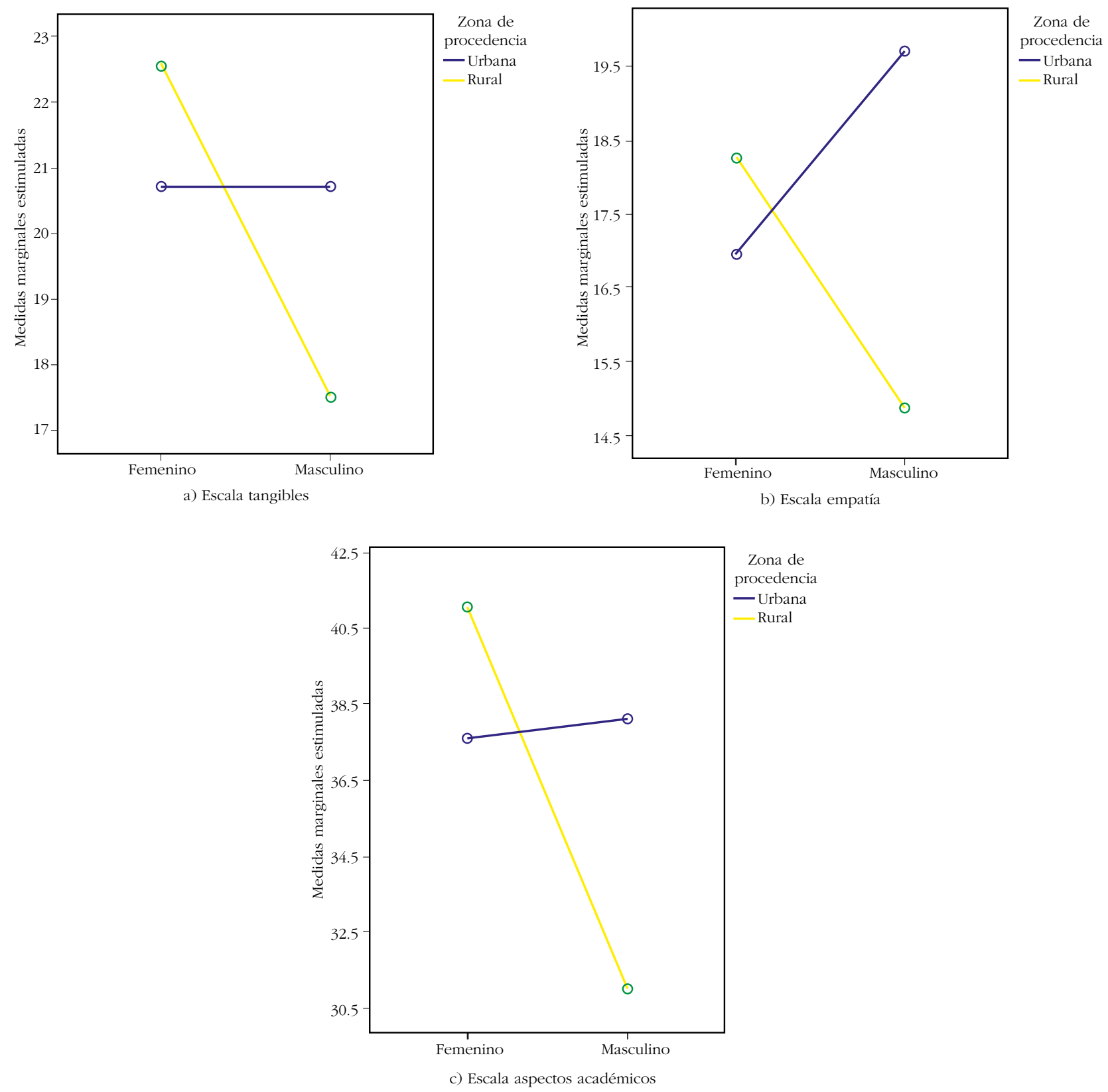

Figura 1. Efecto de moderación producto del sexo y zona de procedencia del estudiantado

Fuente: elaboración del autor. 


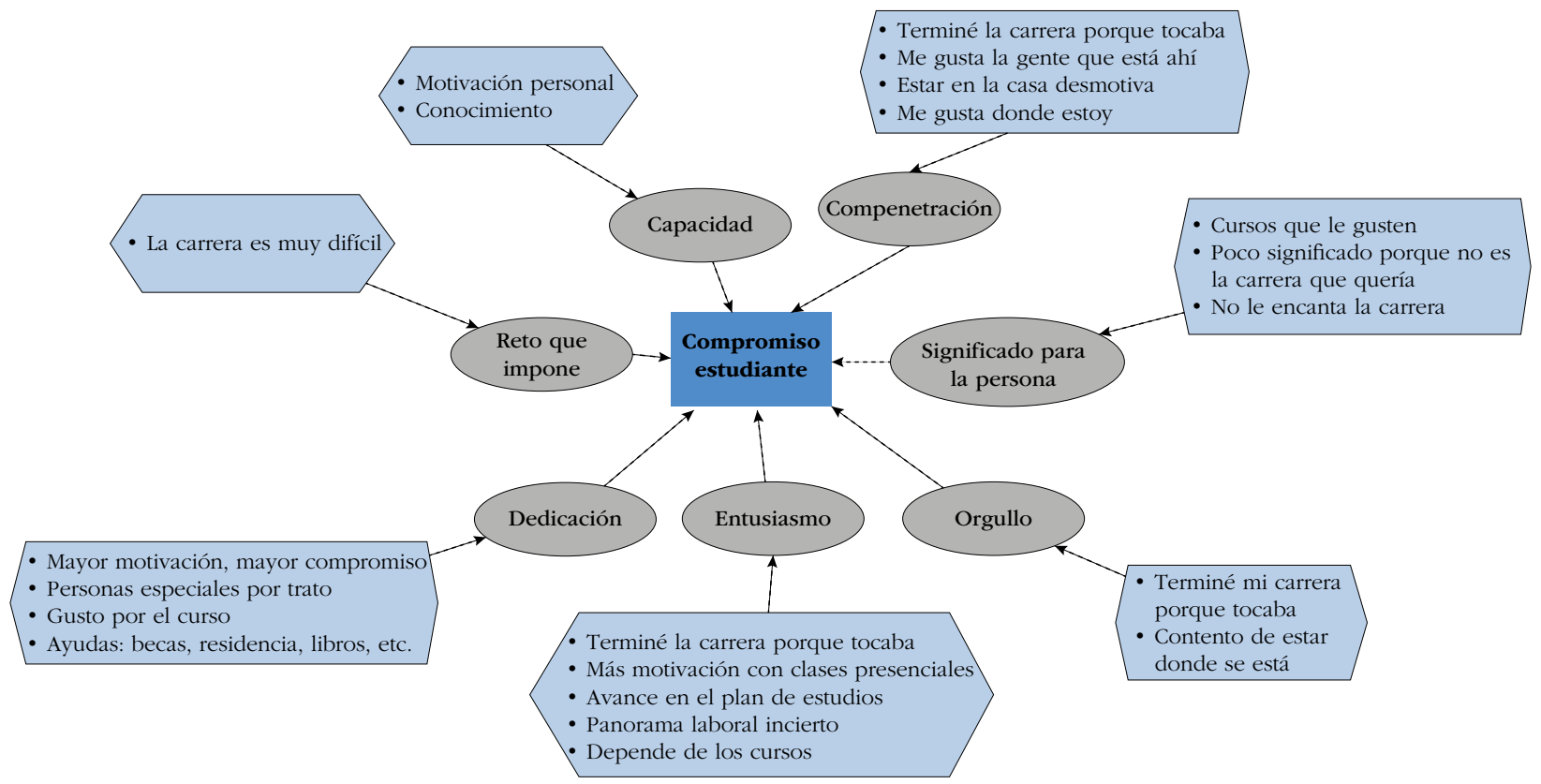

Figura 2. Análisis de los elementos considerados por el estudiantado que tienen impacto en su compromiso

Fuente: elaboración del autor.

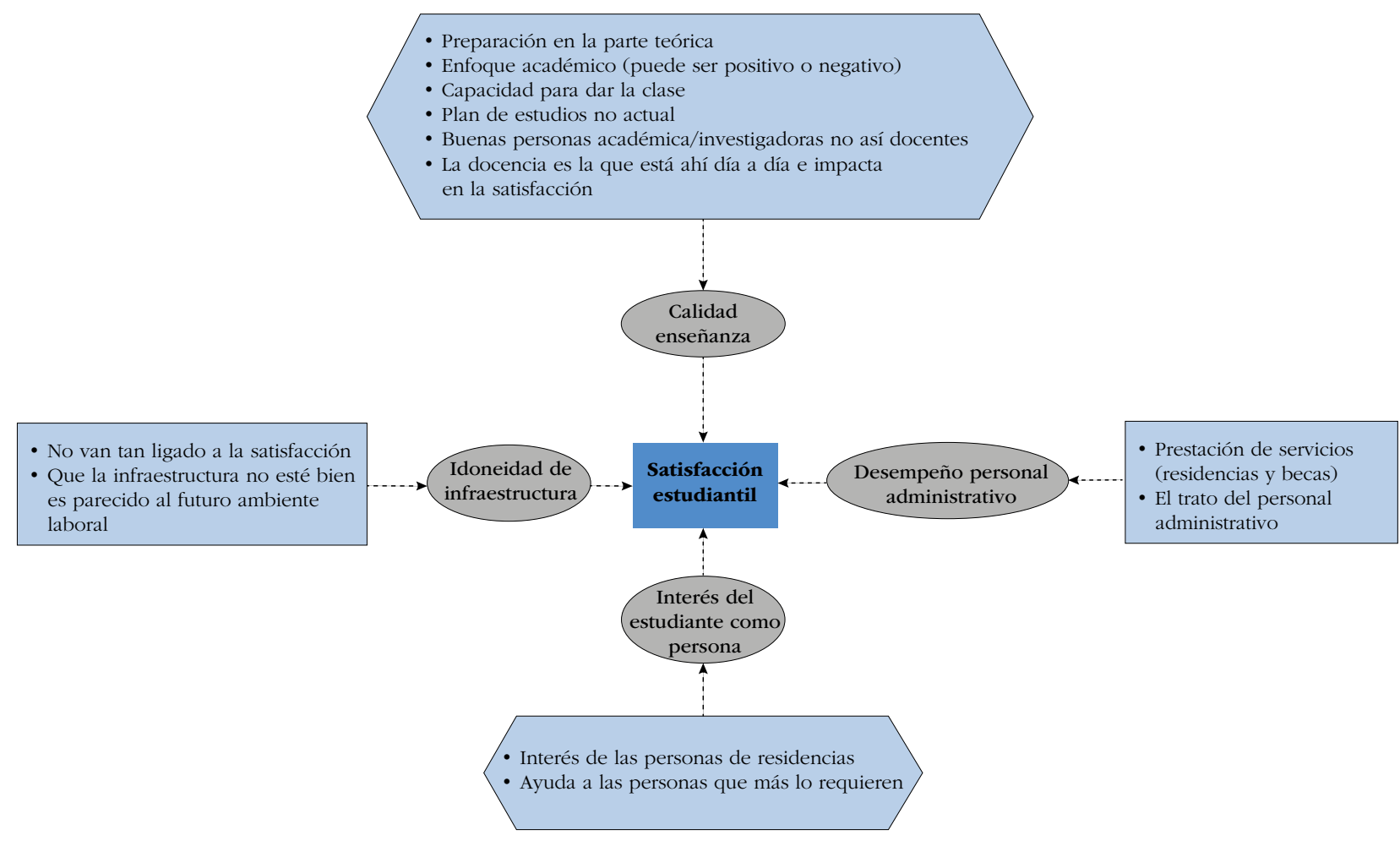

Figura 3. Análisis de los elementos considerados por el estudiantado que tienen impacto en su satisfacción

Fuente: elaboración del autor. 


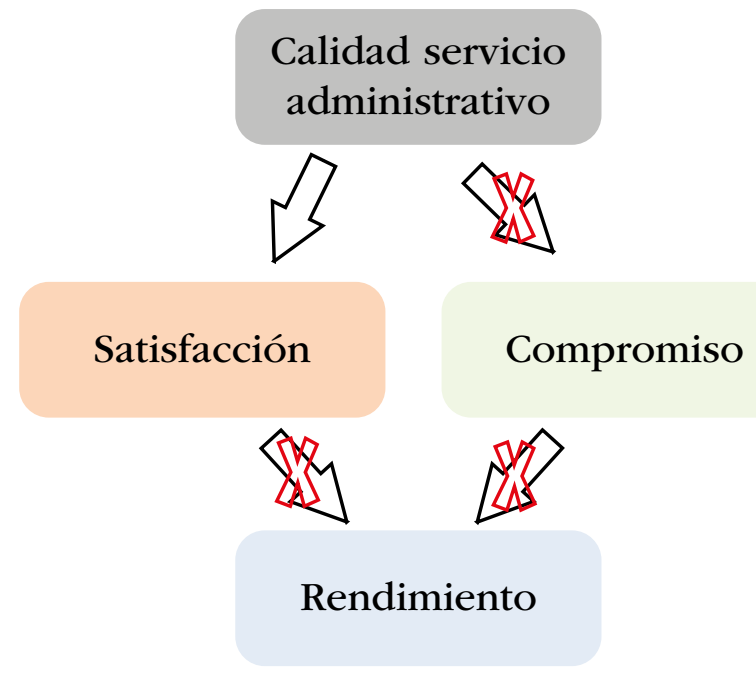

Figura 4. Modelo de interacción de los elementos estudiados a partir de los resultados obtenidos Fuente: elaboración del autor.

Los resultados cuantitativos son explicados por los hallazgos en el componente cualitativo de la investigación. En este sentido, las personas entrevistadas manifiestan de manera reiterada aspectos que tienen que ver con el compromiso personal. Las actividades académicas sin duda son las que tienen impacto directo sobre este componente en el estudiantado. Por ejemplo, una de las personas estudiantes mencionó en el grupo focal: "Yo, por mi convicción personal, no dejaría morir a un curso o perder nunca un curso, o dejaría de lado mis evaluaciones, pero sí que en definitiva, cuando tenía mayor motivación por los cursos estaba, que estaba llevando o uno se compromete más con lo que hace" (estudiante 3, comunicación personal, 17 de junio de 2020).

La tendencia expresada por el estudiantado está en la línea de una motivación interna intrínseca o promovida por factores externos relacionados con su paso por la universidad. Hay una asociación directa entre la motivación que sienten y su nivel de compromiso. Entienden este como el resultado de una serie de factores no directamente asociados al componente académico, incluyen otros ejes del quehacer universitario como disparadores.

Es importante resaltar que el estudiantado no percibe como un reto su labor, ni la carrera que cursa, aunque sí mencionan que estas son difíciles. El orgullo es otro elemento no ubicado dentro de lo manifestado, al contrario, la tendencia es a no tener un reconocimiento por 
lo que estudian, pero sí por la Universidad de Costa Rica y por los grupos de investigación de los cuales forman parte (ver figura 2).

Un aspecto que sí está relacionado con la calidad del servicio y que el estudiantado lo considera como significativo para el compromiso que siente es lo referente a la ayuda socioeconómica y de servicios que brinda la universidad. En este sentido uno de los estudiantes declaró: "Definitivamente lo que viene siendo becas y residencias hace que uno se comprometa un poco más porque es una manera de devolver un poco de lo que le dan a uno" (estudiante 5, comunicación personal, 17 de junio de 2020). No es perceptible que el estudiantado considere la calidad del servicio como un elemento significativo en su compromiso. Se puede observar que solo uno de los aspectos asociados a las subcategorías (dedicación, ver figura 2) está en esta línea, precisamente el mencionado en la cita anterior. Los demás son propios del estudiantado o generados como consecuencia de interacciones con instancias diferentes a las administrativas.

Queda ampliamente constatado que las personas estudiantes centran su satisfacción en su tarea de aprendizaje en la parte académica. Son reiteradas las expresiones que orientaron la conversación a aspectos que tienen que ver con la capacidad del profesorado tanto en su parte de conocimientos como en su capacidad de transmitirlos, como se observa en la figura 3. Sin embargo, la parte académica no se circunscribe a la capacidad del docente, también se hace referencia a asuntos que atañen a la estructura de cada uno de los planes de estudio y a la oferta académica de las escuelas. Una estudiante aseveró en este sentido: "Estoy satisfecha. Sin embargo, no tanto con el plan de estudios. Sí le haría modificaciones al plan de estudios y creo que es una de las cosillas que quizás desmotivan, o yo diría como aspectos negativos" (estudiante 3, comunicación personal, 17 de junio de 2020).

El estudiantado coloca un peso significativo en la parte académica, pero sí es consciente de que la labor administrativa tiene un impacto sobre su satisfacción. No obstante, no logra establecer en qué medida le afecta y lo toma como un componente que impacta marginalmente, contrario a lo encontrado en la parte cuantitativa. Muchas de las observaciones van en la misma línea de la parte académica. La falta de recursos para abrir un curso es una de las más citadas y es vista como un problema académico. 
Las facilidades que brinda la universidad y la atención a la persona estudiante como ser humano tienen un valor para ella y se refleja en su satisfacción. Esto no ocurre con el caso de la idoneidad de la infraestructura, pues no se encuentra relación con la satisfacción. Puede tratarse del efecto de no tener punto de comparación, lo que hace que las condiciones actuales se consideren suficientes, pero no relevantes, para su satisfacción (ver figura 3).

En la fase cuantitativa de este trabajo, los datos no son consistentes con los mostrados anteriormente. En primera instancia, las correlaciones entre las escalas de la calidad y las de la satisfacción tienen asociación positiva en niveles que van desde moderados a altos. Todas las escalas correlacionan entre ellas y esta es significativa. La incongruencia entre los datos cualitativos y cuantitativos es aún mayor cuando se analizan los modelos predictivos. En estos se observa cómo la varianza explicada para las escalas de aspectos administrativos, infraestructura y servicios universitarios y aspectos académicos alcanza hasta un $49 \%$ para la primera, un $41 \%$ para la segunda y un $37 \%$ en la tercera.

A pesar de que se evidencia que existe influencia de la calidad sobre la satisfacción, el efecto no fue observado de manera contundente en el análisis cualitativo. Se puede especular que esto es producto de la invisibilidad que el servicio administrativo puede tener si se presta bien, pero que esta relación sí se puede captar cuando la información se consulta a través de instrumentos independientes, como se hace en los cuestionarios.

Por otra parte, los resultados cuantitativos son contradictorios con lo expresado por las personas estudiantes en la sesión de grupo focal. Estas manifestaban que la calidad del servicio sí se relacionaba con el rendimiento y que podía ser negativo o positivo. Si bien una correlación no demuestra causalidad, era de esperar que existiera asociación entre ellas y no la hay.

En cuanto a los efectos de moderación, solo algunas escalas se vieron influenciadas por las características de sexo y zona de procedencia. Específicamente en la zona rural y con una valoración inferior por parte de los hombres. Estos resultados cuantitativos no pudieron ser constatados ni refutados a partir de la información recabada en la entrevista al estudiantado. Pese a tener una representatividad en ambos factores sociodemográficos, no se indicó en ningún momento que tuviera algún impacto. 


\section{Conclusiones}

A partir de la información recabada y analizada, se puede concluir que las hipótesis $H 1$ y H2 deben ser aceptadas. Existen correlaciones entre las escalas de la calidad del servicio y las escalas de la satisfacción del estudiantado en un nivel moderado. Los coeficientes de Rho de Spearman van desde 0.399 hasta 0.480 . Lo mismo ocurre en el caso de las escalas del compromiso, donde solo la escala de capacidad de respuesta de la calidad del servicio no presenta correlación, aunque en este caso esta es media porque los valores van desde 0.172 a 0.314 . Adicionalmente, es contundente que no existe correlación entre esta calidad y el rendimiento del estudiantado, por lo que la hipótesis $H 3$ se rechaza.

Con respecto a la $\mathrm{H} 4$, se corrobora que la calidad del servicio sí predice la satisfacción del grupo de interés. Las escalas de tangibles, seguridad y empatía logran predecir el $49 \%$ y $43 \%$ de la varianza de las escalas de aspectos administrativos e infraestructura y servicios universitarios de la satisfacción, respectivamente. Por su parte, la escala de tangibles explica el 37\% de la varianza de la escala de aspectos académicos. Por el contrario, un comportamiento predictivo para el compromiso es nulo porque no se encontró un modelo. Ante esto, $H 5$ debe ser rechazada. Lo anterior se repite para el caso de $H 6$, por lo que debe ser rechazada también.

En el caso de las hipótesis 7 y 8, los modelos predictivos tienen una muy pequeña capacidad predictiva del rendimiento ( $3 \%$ en el caso de la satisfacción y $12 \%$ en el del compromiso), por lo que para efectos de este estudio se considera que esta no es significativa y $H 7$ y $H 8$ son rechazadas. En el caso de $H 9$, los resultados muestran que existen efectos de moderación producto de la interacción de las variables sexo y zona de procedencia para dos escalas de la calidad del servicio y una de la satisfacción. Por lo tanto, se acepta esta hipótesis.

De las manifestaciones realizadas por las personas estudiantes, se concluye que suelen confundir la calidad del servicio administrativo con la calidad académica. Es apreciable un sesgo al entender como parte del sistema administrativo al personal docente. La amplia variedad de factores que inciden sobre la satisfacción y compromiso complica la focalización en los que se derivaban del servicio administrativo. El estudiantado entiende su 
satisfacción como una consecuencia, en mayor medida, de lo que la universidad le brinda y de sus logros académicos. No obstante, su nivel de compromiso es dependiente de la capacidad pedagógica que tenga el personal docente y de grupos de apoyo paralelos en los cuales pueda participar (grupos de investigación). Esto deja un margen pequeño para un compromiso intrínseco del estudiantado, sin embargo, se identifica que en ocasiones se acude a convicciones personales para potenciar el compromiso.

En términos generales, se encontró que la calidad del servicio administrativo tiene nulo impacto en el compromiso del estudiantado, pero sí en su satisfacción, por razones que no necesariamente son las que interesaban en este estudio (calidad de la docencia). En lo que no hay discusión es que este grupo no percibe como importantes para su rendimiento el compromiso y satisfacción que experimentan producto de la calidad del servicio administrativo. Consideran que su desempeño se asocia más al compromiso producto de una convicción personal y no por factores externos. Esto llevó a replantear el modelo de interacción propuesto al inicio, donde la calidad del servicio impactaba directamente la satisfacción y el compromiso y estos el rendimiento, por uno donde solo la calidad del servicio y la satisfacción se encuentran relacionadas.

La invisibilidad del servicio administrativo producto de una buena ejecución y la tendencia natural del ser humano a dar mayor relevancia a los aspectos negativos son elementos que dificultan la valoración objetiva de la calidad y de su impacto. Sin embargo, es evidente que el estudiantado percibe como factores de índole más personal los que inciden sobre su nivel de compromiso y rendimiento académico. A través de un buen servicio administrativo será posible mejorar el impacto que ya se da sobre la satisfacción y hacer que los otros dos elementos también sean favorecidos por este. 


\section{Referencias}

Abdullah, F. (2006). Measuring service quality in higher education: HEdPERF versus Servperf. Marketing Intelligence \& Planning, 24(1),31-47.https://doi.org/10.1108/02634500610641543

Abdullah, M. C., Teoh, H. C., Roslan, S., \& Uli, J. (2015). Student engagement: concepts, development and application in Malaysian universities. Journal of Educational and Social Research, 5(2), 275-283. https://doi.org/10.5901/jesr.2015.v5n2p275

Ahdika, A. (2010). Improvement of quality, interest, critical, and analytical thinking ability of students through the application of research based learning (RBL) in introduction to stochastic processes subject. International Electronic Journal of Mathematics Education, 12(2), 167-191.

Ahmed, I., Nawaz, M. M., Ahmad, Z., Ahmad, Z., Shaukat, M. Z., ... Ahmed, N. (2010). Does service quality affect students' performance? Evidence from institutes of higher learning. African Journal of Business Management, 4(12), 2527-2533. Available from https://www. researchgate.net/publication/298145626_Does_service_quality_affect_students'_performance_Evidence_from_institutes_of_higher_learning

Arslanagic-Kalajdzic, M., Čičić, M., \& Kadić-Maglajlić, S. (2014). Students’ perceptions about role of faculty and administrative staff in business education service quality assessment. Tržište, XXVI(junio), 93-108.

Baron, A. (2012). What do engagement measures really mean? Strategic HR Review, 12(1), 2125. https://doi.org/10.1108/14754391311282450

Bayraktaroglu, G., \& Atrek, B. (2010). Testing the superiority and dimensionality of Servqual vs. Servperf in higher education. The Quality Management Journal, 17(1), 47-59. Available from https://www.tandfonline.com/doi/abs/10.1080/10686967.2010.11918260

Buitrago-Suescún, O. Y., Espitia-Cubillos, A. A., \& Mejías-Acosta, A. A. (2017). Análisis de factores para la medición de la satisfacción estudiantil en educación superior: caso ingeniería industrial, Universidad Militar Nueva Granada. Revista Educación en Ingeniería, 12(24), 107-112. https://doi.org/10.26507/rei.v12n24.774

Cadena Badilla, M., Mejías Acosta, A., Vega Robles, A., \& Vásquez Quiroga, J. (2016). La satisfacción estudiantil universitaria: análisis estratégico a partir del análisis de factores. Industrial Data, 18(1), 9. https://doi.org/10.15381/idata.v18i1.12062

Campos, D. F., Dos Santos, G. S., \& Castro, F. N. (2017). Variations in student perceptions of service quality of higher education institutions in Brazil: a longitudinal study. Quality Assurance in Education, 25(4). https://doi.org/10.1108/QAE-02-2016-0008 
Candelas Zamorano, C. O., Gurruchaga Rodríguez, M. E., Mejías Acosta, A., \& Flores Ávila, L. C. (2013). Medición de la satisfacción estudiantil universitaria: un estudio de caso en una institución mexicana. Iberoamerican Journal of Industrial Engineering, 5(9), 261-274.

Çapri, B., Gündüz, B., \& Akbay, S. E. (2017). Utrecht work engagement scale-student forms' (UWES-SF) adaptation to Turkish, validity and reliability studies, and the mediator role of work engagement between academic procrastination and academic responsibility. Kuram ve Uygulamada Egitim Bilimleri, 1 7(2), 411-435. https://doi.org/10.12738/estp.2017.2.0518

Cronin, J. J., \& Taylor, S. A. (1992). Measuring service quality: a reexamination and extension. Journal of Marketing, 56(3), 55-68. https://doi.org/10.2307/1252296

De Miguel, M., \& Arias, J. (1999). La evaluación del rendimiento inmediato en la enseñanza universitaria. Revista de Educación, 320, 353-377.

Duque Oliva, E. J. (2005). Revisión del concepto de calidad del servicio y sus modelos de medición. Innovar, 15(25), 64-80. Recuperado de https://www.redalyc.org/pdf/818/81802505.pdf

Duque Oliva, E. J., \& Diosa Gómez, Y. (2014). Evolución conceptual de los modelos de medición de la percepción de calidad del servicio: una mirada desde la educación superior. Suma de Negocios, 5(12), 180-191. https://doi.org/10.1016/S2215-910X(14)70040-0

Eberle, L., Milan, G. S., \& Dorion, E. (2016). Service quality dimensions and customer satisfaction in a Brazilian university context. Benchmarking: An International Journal, 23(7), 1697-1716. https://doi.org/10.1108/BIJ-09-2014-0089

EsIB-The National Unions of Students in Europe. (2002). European student handbook on quality assurance in higher education. Available from http://www3.uma.pt/jcmarques/docs/ info/qaheducation.pdf

Galeeva, R. B. (2016). Servqual application and adaptation for educational service quality assessments in Russian higher education. Quality Assurance in Education, 24(3), 329-348. https://doi.org/10.1108/QAE-06-2015-0024

Garbanzo, G. M. (2007). Factores asociados al rendimiento académico en estudiantes universitarios, una reflexión desde la calidad de la educación superior pública. Educación, 31(1), 43-63. https://doi.org/10.15517/revedu.v31i1.1252

Great Schools Partnership. (4 de agosto de 2018). Student engagement. Available from http:// edglossary.org/student-engagement/

Hardy, C., \& Bryson, C. (2010). Student engagement: paradigm change or political expediency? Networks Magazine, 9, 19-23. Available from http://arts.brighton.ac.uk/_data/assets/ pdf_file/0011/64775/Christine-Hardy-article-from-Networks09-pp-19-23.pdf

Hemsley-Brown, J., Lowrie, A., Gruber, T., Fuß, S., Voss, R., \& Gläser-Zikuda, M. (2010). Examining student satisfaction with higher education services: using a new measurement 
tool. International Journal of Public Sector Management, 23(2), 105-123. https://doi. org/10.1108/09513551011022474

Hernández Sampieri, R., Fernández Collado, C., \& Baptista Lucio, M. del P. (2010). Metodología de la investigación. Ciudad de México: McGraw-Hill.

Hoyer, R., \& Hoyer, B. (2001). What is quality? Quality Progress, July, 53-62. Available from https://www.researchgate.net/publication/293601798_What_is_quality

IBM. (2015). sPss Statistics (23.0.0.0) [software]. IBM. Available from https://www.ibm.com/analytics/spss-statistics-software

Ipsos MORI. (29 de septiembre de 2018). National Student Survey 2017. Available from http:// www.thestudentsurvey.com/

International Organization for Standardization (Iso). (4 de agosto de 2020). Iso 10001:2007(es). Available from https://www.iso.org/obp/ui/\#iso:std:iso:10001:ed-1:v1:es

Kahu, E. R. (2013). Framing student engagement in higher education. Studies in Higher Education, 38(5), 758-773. https://doi.org/10.1080/03075079.2011.598505

Krause, K.-L., \& Coates, H. (2008). Students' engagement in first-year university. Assessment $\mathcal{E}$ Evaluation in Higher Education, 33(5), 493-505. https://doi.org/10.1080/02602930701698892

Kuh, G. (2009). The National Survey of Student Engagement: conceptual and empirical foundations. New Directions for Institutional Research, 141, 1-11. https://doi.org/10.1002/ir.283

Leeds Trinity University. (5 de agosto de 2018). Student engagement strategy 2015-19. Available from http://www.leedstrinity.ac.uk/KeyDocuments/StudentEngagement Strategy.pdf

Loureiro, S. M. C., \& González, F. J. M. (2012). Duaqual: calidad percibida por docentes y alumnos en la gestión universitaria. Cuadernos de Gestión, 12(1), 107-122. https://doi. org/10.5295/cdg.100251sc

Lupo, T. (2013). A fuzzy Servqual based method for reliable measurements of education quality in Italian higher education area. Expert Systems with Applications, 40(17), 7096-7110. https://doi.org/10.1016/j.eswa.2013.06.045

Matsumoto Nishizawa, R. (2014). Desarrollo del modelo Servqual para la medición de la calidad del servicio en la empresa de publicidad Ayuda Experto. Revista Perspectivas, 34, 181-209. Recuperado de http://www.scielo.org.bo/scielo.php?script=sci_arttext\&pi$\mathrm{d}=$ S1994-37332014000200005\&lng=es\&nrm=iso\&tlng=es

Mejías, A., \& Martínez, D. (2009). Desarrollo de un instrumento para medir la satisfacción estudiantil en educación superior. Docencia Universitaria, 10(2), 29-47. Recuperado de http://saber.ucv.ve/ojs/index.php/rev_docu/article/view/3704 
Morales Sánchez, V., \& Hernández Mendo, A. (2004). Calidad y satisfacción en los servicios: conceptualización. Revista Digital, 10(73), 1-13. Recuperado de http://www.efdeportes. com/efd73/calidad.htm

National Survey of Student Engagement (NSSE). ( $1^{\circ}$ de agosto de 2018). About NSSE. Available from https://nsse.indiana.edu/nsse/about-nsse/index.html

Navarro, M. M., Iglesias, M. P., \& Torres, P. R. (2005). A new management element for universities: satisfaction with the offered courses. International Journal of Educational Management, 19(6), 505-526. https://doi.org/10.1108/09513540510617454

Newmann, F. M., Marks, H. M., \& Gamoran, A. (1996). Authentic pedagogy and student performance. American Journal of Education, 104(August), 280-312. https://doi.org/https:// www.jstor.org/stable/1085433

Olson, A., \& Peterson, R. (5 de agosto de 2018). Student engagement. Strategy brief. Available from https://k12engagement.unl.edu/strategy-briefs/Student Engagement 4-20-15_0.pdf

Parasuraman, A., Zeithaml, V. A., \& Berry, L. L. (1985). A conceptual model of service quality and its implications for future research. Journal of Marketing, 49(4), 41-50. https://doi. org/https://doi.org/10.2307/1251430

Parasuraman, A., Zeithaml, V. A., \& Berry, L. L. (1988). Serqual: a multiple-item scale for measuring consumer perceptions of service quality. Journal of Retailing, 64(1), 12-40. Available from https://www.researchgate.net/publication/200827786_SERvQUAL_A_Multipleitem_Scale_for_Measuring_Consumer_Perceptions_of_Service_Quality

Pereira Puga, M. (2014). Educación superior universitaria: calidad percibida y satisfacción de los egresados (Tesis de doctorado, Departamento de Sociología, Ciencia Política y de la Administración, Universidade da Coruña, La Coruña, España). Recuperado de http:// ruc.udc.es/dspace/bitstream/handle/2183/12349/PereiraPuga_Manuel_TD_2014.pdf?sequence $=2$

Pérez Serrano, G. (1986). Crítica al concepto de rendimiento académico. Revista Española de Pedagogía, 44(174), 521-534.

Pineda Báez, C., Bermúdez Aponte, J. J., Rubiano Bello, Á., Pava García, N., Suárez García, R., \& Cruz Becerra, F. (2014). Compromiso estudiantil y desempeño académico en el contexto universitario colombiano. Revista Electrónica de Investigación y Evaluación Educativa, 2O(2), 1-19. https://doi.org/10.7203/relieve.20.2.4238

Reinoso Sánchez, M. Á. (2009). El análisis matemático aplicado al cálculo de la muestra. Ciencia UNEMI, setiembre, 40-45.

Rezaei, S., Karami Matin, B., Hajizadeh, M., Soroush, A., Mohammadi, Z., ... Jamshidi, K. (2017). Evaluating service quality in the higher education sector in Iran: an examination 
of students' perspective. International Journal of Human Rights in Healthcare, 10(2), 146-155. https://doi.org/10.1108/IJHRH-12-2016-0024

Römer, J. (2016). The Korean utrecht work engagement scale-student (uwEs-s): a factor validation study. TPM - Testing, Psychometrics, Methodology in Applied Psychology, 23(1), 65-81. https://doi.org/10.4473/TPM23.1.5

Salgado, C. (2013). El rendimiento académico y el compromiso de los alumnos que trabajan de la Licenciatura en Informática de la FCA de la UABC. Revista Iberoamericana para la Investigación y el Desarrollo Educativo, julio, 1-16. Recuperado de http://1-11.ride.org. $\mathrm{mx} /$ index.php/RIDESECUNDARIO/article/viewFile/625/612

Schaufeli, W., \& Bakker, A. (2003). The Utrecht Work Engagement Scale: preliminary manual. Available from https://www.researchgate.net/publication/312805574_The_Utrecht_Work_ Engagement_Scale_uwEs_Test_manual

Schaufeli, W., Bakker, A., \& Salanova, M. (2006). The measurement of short questionnaire: a cross-national study. Educational and Psychological Measurement, 66(4), 701-716. https:// doi.org/10.1177/0013164405282471

Schaufeli, W., Salanova, M., Bakker, A., \& González-Romá, V. (2002). The measurement of engagement and burnout: a two sample confirmatory factor analytic approach. Journal of Happiness Studies, 3, 71-92.

Scientific Software Development GmbH. (2019). Atlas.ti (8.4.24.0) [software]. Scientific Software Development GmbH. Available from https://atlasti.com/es/

Seid, G. (2016). Procedimientos para el análisis cualitativo de entrevistas. Una propuesta didáctica. Conferencia presentada en el V Encuentro Latinoamericano de Metodología de las Ciencias Sociales (ецмесs), Mendoza, Argentina. Recuperado de http://elmecs.fahce. unlp.edu.ar/v-elmecs/actas-2016/Seid.pdf/view

Starr, A., Betz, E., \& Menne, J. (1971). College Student Satisfaction Questionnaire (cssQ) manual. Central Iowa Associates, Inc.

Streiner, D. L. (2003). Starting at the beginning: an introduction to coefficient alpha and internal consistency. Journal of Personality Assessment, 80(1), 99-103. https://doi.org/https:// doi.org/10.1207/S15327752JPA8001_18

Taber, K. S. (2017). The use of Cronbach's alpha when developing and reporting research instruments in science education. Research in Science Education, 48(6), 1273-1296. https:// doi.org/10.1007/s11165-016-9602-2

Teas, R. K. (1993). Expectations, performance evaluation, and consumers' perceptions of quality. Journal of Marketing, 57(4), 18-34. https://doi.org/10.2307/1252216 
Torres, E., \& Araya, L. (2010). Construcción de una escala para medir la calidad del servicio de las universidades: una aplicación al contexto chileno. Revista de Ciencias Sociales, 16(1), 54-67. https://doi.org/https://doi.org/10.31876/rcs.v16i1.25484

Trowler, V. (2010). Student engagement literature review. The Higher Education Academy. Available from https://www.advance-he.ac.uk/knowledge-hub/student-engagement-literature-review

Tumino, M. C., \& Poitevin, E. R. (2014). Evaluación de la calidad de servicio universitario desde la percepción de estudiantes y docentes: caso de estudio. Reice. Revista Iberoamericana sobre Calidad, 12(2), 63-84. Recuperado de https://revistas.uam.es/reice/article/view/2856/

Velandia, F., Ardón, N., \& Jara, M. I. (2008). Satisfacción y calidad: análisis de la equivalencia o no de los términos. Revista Gerencia y Salud, 6(13), 139-168. 


\section{Anexo 1. Cuestionario empleado para valorar la calidad percibida por el estudiantado (Servperf)}

Instrucciones: el cuestionario Servperf que se le presenta contiene una serie de afirmaciones relativas a lo que usted piensa sobre aspectos relacionados con las instalaciones y la labor del personal administrativo de la Universidad de Costa Rica. Por favor, seleccione un valor de 1 a 7 en cada afirmación según lo que usted considera es la situación actual (percepción) en cada uno de los ítems.

\begin{tabular}{|c|c|c|c|c|c|c|c|}
\hline \multirow[b]{2}{*}{ 1. El equipo para educación es actual y moderno } & \multicolumn{3}{|c|}{$\begin{array}{l}\text { Totalmente } \\
\text { en desacuerdo }\end{array}$} & \multirow[b]{2}{*}{4} & \multirow[b]{2}{*}{5} & \multicolumn{2}{|c|}{$\begin{array}{l}\text { Totalmente } \\
\text { de acuerdo }\end{array}$} \\
\hline & 1 & 2 & 3 & & & 6 & 7 \\
\hline $\begin{array}{l}\text { 2. La cantidad de equipo para educación e infraestructu- } \\
\text { ra es apropiada }\end{array}$ & 1 & 2 & 3 & 4 & 5 & 6 & 7 \\
\hline $\begin{array}{l}\text { 3. La apariencia personal de las personas dedicadas a las } \\
\text { labores administrativas es apropiada según sus funciones }\end{array}$ & 1 & 2 & 3 & 4 & 5 & 6 & 7 \\
\hline 4. El personal administrativo es entusiasta y amigable & 1 & 2 & 3 & 4 & 5 & 6 & 7 \\
\hline $\begin{array}{l}\text { 5. El personal administrativo presenta interés en resolver } \\
\text { los problemas de los/las estudiantes }\end{array}$ & 1 & 2 & 3 & 4 & 5 & 6 & 7 \\
\hline $\begin{array}{l}\text { 6. Al solicitar ayuda, el personal administrativo es servicial } \\
\text { y cortés }\end{array}$ & 1 & 2 & 3 & 4 & 5 & 6 & 7 \\
\hline $\begin{array}{l}\text { 7. El personal administrativo le suministra la información } \\
\text { adecuada cuando el/la estudiante lo requiere }\end{array}$ & 1 & 2 & 3 & 4 & 5 & 6 & 7 \\
\hline $\begin{array}{l}\text { 8. El personal administrativo puede responder las pre- } \\
\text { guntas de los/las estudiantes adecuadamente }\end{array}$ & 1 & 2 & 3 & 4 & 5 & 6 & 7 \\
\hline $\begin{array}{l}\text { 9. El personal administrativo está disponible a las consul- } \\
\text { tas de los/las estudiantes en los horarios establecidos }\end{array}$ & 1 & 2 & 3 & 4 & 5 & 6 & 7 \\
\hline $\begin{array}{l}\text { 10. La administración maneja los registros educativos de } \\
\text { los/las estudiantes correctamente }\end{array}$ & 1 & 2 & 3 & 4 & 5 & 6 & 7 \\
\hline $\begin{array}{l}\text { 11. El personal administrativo conoce las políticas, regu- } \\
\text { laciones y pautas para responder las preguntas de los/las } \\
\text { estudiantes }\end{array}$ & 1 & 2 & 3 & 4 & 5 & 6 & 7 \\
\hline $\begin{array}{l}\text { 12. El personal administrativo tiene los conocimientos } \\
\text { necesarios para proporcionar los servicios que requiere } \\
\text { el/la estudiante }\end{array}$ & 1 & 2 & 3 & 4 & 5 & 6 & 7 \\
\hline $\begin{array}{l}\text { 13. El personal administrativo tiene las habilidades para } \\
\text { cumplir con sus responsabilidades }\end{array}$ & 1 & 2 & 3 & 4 & 5 & 6 & 7 \\
\hline $\begin{array}{l}\text { 14. Los servicios que presta el personal administrativo } \\
\text { se realizan con eficiencia de tal forma que no requiere } \\
\text { seguimiento de los/las estudiantes }\end{array}$ & 1 & 2 & 3 & 4 & 5 & 6 & 7 \\
\hline $\begin{array}{l}\text { 15. El personal administrativo presta atención a los senti- } \\
\text { mientos de los/las estudiantes }\end{array}$ & 1 & 2 & 3 & 4 & 5 & 6 & 7 \\
\hline
\end{tabular}




\begin{tabular}{|c|c|c|c|c|c|c|c|}
\hline \multirow{2}{*}{$\begin{array}{l}\text { Ítem } \\
\qquad \begin{array}{l}\text { 16. El personal administrativo respeta las opiniones y } \\
\text { sugerencias de los/las estudiantes }\end{array}\end{array}$} & \multicolumn{5}{|c|}{$\begin{array}{l}\text { Totalmente } \\
\text { en desacuerdo }\end{array}$} & \multicolumn{2}{|c|}{$\begin{array}{l}\text { Totalmente } \\
\text { de acuerdo }\end{array}$} \\
\hline & 1 & 2 & 3 & 4 & 5 & 6 & 7 \\
\hline $\begin{array}{l}\text { 17. El personal administrativo escucha con entusiasmo las } \\
\text { opiniones de los/las estudiantes }\end{array}$ & 1 & 2 & 3 & 4 & 5 & 6 & 7 \\
\hline $\begin{array}{l}\text { 18. El personal administrativo explica con paciencia las } \\
\text { políticas, los reglamentos y las pautas de la universidad }\end{array}$ & 1 & 2 & 3 & 4 & 5 & 6 & 7 \\
\hline $\begin{array}{l}\text { 19. El personal administrativo provee los servicios a los/ } \\
\text { las estudiantes de manera oportuna }\end{array}$ & 1 & 2 & 3 & 4 & 5 & 6 & 7 \\
\hline $\begin{array}{l}\text { 20. El personal administrativo brinda servicios a los/las } \\
\text { estudiantes sin ningún error }\end{array}$ & 1 & 2 & 3 & 4 & 5 & 6 & 7 \\
\hline $\begin{array}{l}\text { 21. El personal administrativo brinda servicios a los/las } \\
\text { estudiantes de manera equitativa y justa }\end{array}$ & 1 & 2 & 3 & 4 & 5 & 6 & 7 \\
\hline $\begin{array}{l}\text { 22. Los/las estudiantes no tienen que esperar mucho } \\
\text { tiempo por los servicios que necesitan }\end{array}$ & 1 & 2 & 3 & 4 & 5 & 6 & 7 \\
\hline
\end{tabular}




\section{Anexo 2. Cuestionario empleado para valorar el compromiso del estudiantado (UWES)}

Instrucciones: las siguientes afirmaciones se refieren a los sentimientos de las personas cuando realizan actividades como asistir a clases teóricas y prácticas, ir a la biblioteca, hacer trabajos en grupo, estudiar, etc. Por favor, lea cuidadosamente cada pregunta y decida si se ha sentido de esta forma. Si nunca se ha sentido así, conteste '0' (cero); y, en caso contrario, indique qué tan reiteradamente se ha sentido así teniendo en cuenta la siguiente escala de respuesta (de 1 a 6 ).

\begin{tabular}{|c|c|c|c|c|c|c|c|}
\hline Ítem & Nunca & & & & & & Siempre \\
\hline $\begin{array}{l}\text { 1. Mis tareas como estudiante me hacen sentir lleno(a) } \\
\text { de energía }\end{array}$ & 0 & 1 & 2 & 3 & 4 & 5 & 6 \\
\hline 2. Creo que mi carrera tiene significado & 0 & 1 & 2 & 3 & 4 & 5 & 6 \\
\hline $\begin{array}{l}\text { 3. El tiempo "pasa volando" cuando realizo mis tareas } \\
\text { como estudiante }\end{array}$ & 0 & 1 & 2 & 3 & 4 & 5 & 6 \\
\hline $\begin{array}{l}\text { 4. Me siento fuerte y vigoroso(a) cuando estoy estu- } \\
\text { diando o voy a las clases }\end{array}$ & 0 & 1 & 2 & 3 & 4 & 5 & 6 \\
\hline 5. Estoy entusiasmado(a) con mi carrera & 0 & 1 & 2 & 3 & 4 & 5 & 6 \\
\hline $\begin{array}{l}\text { 6. Olvido todo lo que pasa alrededor de mí cuando } \\
\text { estoy abstraído(a) con mis estudios }\end{array}$ & 0 & 1 & 2 & 3 & 4 & 5 & 6 \\
\hline 7. Mis estudios me inspiran cosas nuevas & 0 & 1 & 2 & 3 & 4 & 5 & 6 \\
\hline $\begin{array}{l}\text { 8. Cuando me levanto por la mañana me apetece ir a } \\
\text { clase o estudiar }\end{array}$ & 0 & 1 & 2 & 3 & 4 & 5 & 6 \\
\hline $\begin{array}{l}\text { 9. Soy feliz cuando estoy haciendo tareas relacionadas } \\
\text { con mis estudios }\end{array}$ & 0 & 1 & 2 & 3 & 4 & 5 & 6 \\
\hline 10. Estoy orgulloso(a) de hacer esta carrera & 0 & 1 & 2 & 3 & 4 & 5 & 6 \\
\hline 11. Estoy inmerso(a) en mis estudios & 0 & 1 & 2 & 3 & 4 & 5 & 6 \\
\hline $\begin{array}{l}\text { 12. Puedo seguir estudiando durante largos períodos } \\
\text { de tiempo }\end{array}$ & 0 & 1 & 2 & 3 & 4 & 5 & 6 \\
\hline 13. Mi carrera es retadora para mí & 0 & 1 & 2 & 3 & 4 & 5 & 6 \\
\hline $\begin{array}{l}\text { 14. Me "dejo llevar" cuando realizo mis tareas como } \\
\text { estudiante }\end{array}$ & 0 & 1 & 2 & 3 & 4 & 5 & 6 \\
\hline $\begin{array}{l}\text { 15. Soy muy "resistente" para afrontar mis tareas como } \\
\text { estudiante }\end{array}$ & 0 & 1 & 2 & 3 & 4 & 5 & 6 \\
\hline 16. Es difícil para mí separarme de mis estudios & 0 & 1 & 2 & 3 & 4 & 5 & 6 \\
\hline $\begin{array}{l}\text { 17. En mis tareas como estudiante no paro incluso si no } \\
\text { me encuentro bien }\end{array}$ & 0 & 1 & 2 & 3 & 4 & 5 & 6 \\
\hline
\end{tabular}




\section{Anexo 3. Cuestionario empleado para valorar la satisfacción del estudiantado (SEU)}

Instrucciones: las siguientes afirmaciones se refieren a las percepciones de las personas cuando hacen uso de los servicios o instalaciones universitarias. Lea cuidadosamente cada una y decida su nivel de acuerdo con el enunciado presentado. Por favor, seleccione un valor de 1 a 7 en cada afirmación según lo que usted considera es su satisfacción en cuanto al cumplimiento de sus necesidades, expectativas y requisitos en cada uno de los ítems.

\begin{tabular}{|c|c|c|c|c|c|c|c|}
\hline \multirow{2}{*}{$\begin{array}{l}\text { Ítem } \\
\begin{array}{l}\text { 1. Mis profesores(as) demuestran dominio acerca de la } \\
\text { materia que imparten }\end{array}\end{array}$} & \multicolumn{3}{|c|}{$\begin{array}{l}\text { Totalmente } \\
\text { en desacuerdo }\end{array}$} & \multirow[b]{2}{*}{4} & \multirow[b]{2}{*}{5} & \multicolumn{2}{|c|}{$\begin{array}{l}\text { Totalmente } \\
\text { de acuerdo }\end{array}$} \\
\hline & 1 & 2 & 3 & & & 6 & 7 \\
\hline $\begin{array}{l}\text { 2. Me encuentro satisfecho(a) con el liderazgo de los } \\
\text { profesores }\end{array}$ & 1 & 2 & 3 & 4 & 5 & 6 & 7 \\
\hline $\begin{array}{l}\text { 3. Mis profesores(as) me están preparando para compor- } \\
\text { tarme con autonomía e iniciativa }\end{array}$ & 1 & 2 & 3 & 4 & 5 & 6 & 7 \\
\hline 4. Mis profesores(as) me enseñan a trabajar en equipo & 1 & 2 & 3 & 4 & 5 & 6 & 7 \\
\hline $\begin{array}{l}\text { 5. Mis profesores(as) me están capacitando para expre- } \\
\text { sarme en público con seguridad y lenguaje apropiado }\end{array}$ & 1 & 2 & 3 & 4 & 5 & 6 & 7 \\
\hline $\begin{array}{l}\text { 6. La Dirección de la institución se preocupa por el bien- } \\
\text { estar de nosotros(as) los(as) estudiantes }\end{array}$ & 1 & 2 & 3 & 4 & 5 & 6 & 7 \\
\hline $\begin{array}{l}\text { 7. Me encuentro satisfecho(a) con los contenidos técni- } \\
\text { cos y metodológicos de las materias }\end{array}$ & 1 & 2 & 3 & 4 & 5 & 6 & 7 \\
\hline $\begin{array}{l}\text { 8. La biblioteca ofrece recursos bibliográficos que permi- } \\
\text { ten cumplir con mis actividades académicas }\end{array}$ & 1 & 2 & 3 & 4 & 5 & 6 & 7 \\
\hline $\begin{array}{l}\text { 9. Cuando estoy dentro de las instalaciones de la institu- } \\
\text { ción me encuentro seguro(a) }\end{array}$ & 1 & 2 & 3 & 4 & 5 & 6 & 7 \\
\hline $\begin{array}{l}\text { 10. La mayoría de mis profesores(as) me trata con respe- } \\
\text { to y profesionalismo }\end{array}$ & 1 & 2 & 3 & 4 & 5 & 6 & 7 \\
\hline $\begin{array}{l}\text { 11. La programación de clases (horarios, secciones y } \\
\text { ofertas de estas) me permite completar mis estudios en } \\
\text { un tiempo razonable }\end{array}$ & 1 & 2 & 3 & 4 & 5 & 6 & 7 \\
\hline $\begin{array}{l}\text { 12. Las evaluaciones (notas de exámenes, trabajos } \\
\text { escritos u orales, y otras) que me realizan los(as) profe- } \\
\text { sores(as) miden el nivel de la labor académica que he } \\
\text { realizado }\end{array}$ & 1 & 2 & 3 & 4 & 5 & 6 & 7 \\
\hline $\begin{array}{l}\text { 13. Los laboratorios cuentan con los equipos y materiales } \\
\text { necesarios }\end{array}$ & 1 & 2 & 3 & 4 & 5 & 6 & 7 \\
\hline $\begin{array}{l}\text { 14. El personal administrativo se preocupa por darme } \\
\text { una excelente atención }\end{array}$ & 1 & 2 & 3 & 4 & 5 & 6 & 7 \\
\hline
\end{tabular}




\begin{tabular}{|c|c|c|c|c|c|c|c|}
\hline \multirow[b]{2}{*}{$\begin{array}{l}\text { 15. Las condiciones sanitarias de las instalaciones son } \\
\text { adecuadas }\end{array}$} & \multicolumn{5}{|c|}{$\begin{array}{l}\text { Totalmente } \\
\text { en desacuerdo }\end{array}$} & \multicolumn{2}{|c|}{$\begin{array}{l}\text { Totalmente } \\
\text { de acuerdo }\end{array}$} \\
\hline & 1 & 2 & 3 & 4 & 5 & 6 & 7 \\
\hline 16. El proceso de matrícula es rápido, sencillo y cómodo & 1 & 2 & 3 & 4 & 5 & 6 & 7 \\
\hline $\begin{array}{l}\text { 17. Las aulas de clase son cómodas y adecuadas para } \\
\text { concentrarme en mis estudios }\end{array}$ & 1 & 2 & 3 & 4 & 5 & 6 & 7 \\
\hline $\begin{array}{l}\text { 18. En las sodas de la institución se encuentra comida } \\
\text { balanceada y a un precio accesible }\end{array}$ & 1 & 2 & 3 & 4 & 5 & 6 & 7 \\
\hline $\begin{array}{l}\text { 19. La institución me brinda experiencias prácticas, fuera } \\
\text { del aula, que me capacitan para el mundo del trabajo }\end{array}$ & 1 & 2 & 3 & 4 & 5 & 6 & 7 \\
\hline
\end{tabular}

